\title{
PLUME-MoM 1.0: A new integral model of volcanic plumes based on the method of moments
}

\author{
M. de' Michieli Vitturi ${ }^{1}$, A. Neri ${ }^{1}$, and S. Barsotti ${ }^{2}$ \\ ${ }^{1}$ Istituto Nazionale di Geofisica e Vulcanologia, Pisa, Italy \\ ${ }^{2}$ Icelandic Meteorological Office, Reykjavík, Iceland \\ Correspondence to: M. de’ Michieli Vitturi (mattia.demichielivitturi@ingv.it) \\ Received: 27 March 2015 - Published in Geosci. Model Dev. Discuss.: 5 May 2015 \\ Revised: 24 July 2015 - Accepted: 28 July 2015 - Published: 6 August 2015
}

\begin{abstract}
In this paper a new integral mathematical model for volcanic plumes, named PLUME-MoM, is presented. The model describes the steady-state dynamics of a plume in a 3-D coordinate system, accounting for continuous variability in particle size distribution of the pyroclastic mixture ejected at the vent. Volcanic plumes are composed of pyroclastic particles of many different sizes ranging from a few microns up to several centimeters and more. A proper description of such a multi-particle nature is crucial when quantifying changes in grain-size distribution along the plume and, therefore, for better characterization of source conditions of ash dispersal models. The new model is based on the method of moments, which allows for a description of the pyroclastic mixture dynamics not only in the spatial domain but also in the space of parameters of the continuous size distribution of the particles. This is achieved by formulation of fundamental transport equations for the multi-particle mixture with respect to the different moments of the grainsize distribution. Different formulations, in terms of the distribution of the particle number, as well as of the mass distribution expressed in terms of the Krumbein log scale, are also derived. Comparison between the new moments-based formulation and the classical approach, based on the discretization of the mixture in $N$ discrete phases, shows that the new model allows for the same results to be obtained with a significantly lower computational cost (particularly when a large number of discrete phases is adopted). Application of the new model, coupled with uncertainty quantification and global sensitivity analyses, enables the investigation of the response of four key output variables (mean and standard deviation of the grain-size distribution at the top of the plume, plume height and amount of mass lost by the plume
\end{abstract}

during the ascent) to changes in the main input parameters (mean and standard deviation) characterizing the pyroclastic mixture at the base of the plume. Results show that, for the range of parameters investigated and without considering interparticle processes such as aggregation or comminution, the grain-size distribution at the top of the plume is remarkably similar to that at the base and that the plume height is only weakly affected by the parameters of the grain distribution. The adopted approach can be potentially extended to the consideration of key particle-particle effects occurring in the plume including particle aggregation and fragmentation.

\section{Introduction}

In the past decades, numerical simulation of volcanic eruptions has greatly advanced and models are now often able to deal with the multi-phase nature of volcanic flows. This is the case, for example, with models describing the dynamics of pyroclastic particles in a volcanic plume, or that of bubbles and crystals dispersed in the magma rising in a volcanic conduit. Despite this, in numerical models, the polydispersity associated with the multi-phase nature of volcanic flows is often ignored or largely simplified (Valentine and Wohletz, 1989; Neri at al., 2003; Dartevelle, 2004; Dufek and Bergantz, 2007; Esposti Ongaro et al., 2007; de' Michieli Vitturi et al., 2010). For instance, in most of the existing conduit models, crystals and bubbles are treated as simple flow components and described by volume fractions only, while in plume dynamics and ash dispersal models the grain-size distribution (GSD) of pyroclasts is discretized in a finite number of classes (i.e., phases). Both approaches make proper 
treatment of the continuous variability of the dimension of pyroclastic particles and gas bubbles difficult. Literature results (Llewellin et al., 2002; Pal, 2003; Costa et al., 2010) clearly show that this variability can largely affect relevant physical/chemical processes that occur during the transport of the dispersed phase such as, for example, the nucleation and growth of bubbles and the coalescence/breakage of bubbles and crystals in the conduit or the aggregation of pyroclastic particles in a volcanic plume.

A theoretical framework and the corresponding computational models, namely, the method of moments for disperse multi-phase flows, have been developed in the past decades, mostly in the chemical engineering community (Hulburt and Katz, 1964; Marchisio et al., 2003), to track the evolution of these systems not only in the physical space but also in the space of properties of the dispersed phase (called internal coordinates). According to this method, a population balance equation is formulated as a continuity statement written in terms of a density function. From the density function some integral quantities of interest (namely, the moments, i.e., specific quantitative measures of the shape of the density function) are then derived and their transport equations are formulated.

In this work we present an extension of the Eulerian steady-state volcanic plume model presented in Barsotti et al. (2008) (derived from Bursik, 2001) obtained by adopting the method of moments. In contrast to the original works where pyroclastic particles are partitioned into a finite number of classes with different sizes and properties, the new model is able to consider a continuous size distribution function of pyroclasts, $f(D)$, representing the number or the mass fraction of particles (per unit volume) with diameter between $D$ and $D+d D$. Accordingly, conservation equations of the plume are expressed in terms of the transport equations for the moments of the ash particle size distribution. In particular, in the following we present the new multi-phase model formulation based on the implementation of the quadrature method of moments (McGraw, 2006) and we investigate the sensitivity of the model to uncertain or variable input parameters such as those describing the grain-size distribution of the mixture. To quantify and incorporate this epistemic uncertainty affecting the input parameters (characterizing lack-of-knowledge) into our application of the model, we tested two different approaches, a modification of the Monte Carlo method based on Latin hypercube sampling (LHS) and a stochastic approach, namely, the generalized polynomial chaos expansion (gPCE) method.

This paper is organized as follows: in Sect. 2 we present the method of moments applied to two different descriptions of particle distribution. In Sect. 3 the equations of the model for the two formulations are described. Section 4 is devoted to the numerical discretization of the model and the numerical implementation of the method of moments. Section 5 presents the application of the model to three test cases with a comparison of the model results for different formulations of the plume model, and finally an uncertainty quantification and a sensitivity analysis are applied to model results.

\section{Method of moments}

\subsection{Moments of the size distribution}

In contrast to previous works, where the solid particles are partitioned in a finite number of classes with different sizes (Barsotti et al., 2008), we introduce here a continuous size distribution function representing the number (or mass) concentration of particles (per unit volume) as a function of particle diameter. In general, this particle size distribution (PSD) is a function of time $t$, of the spatial coordinate and of the diameter of the particles.

First, we present the method of moments for a particle size distribution $f(D)$, representing the number concentration of particles (particles per unit volume) with diameter between $D$ and $D+d D$, where $D$ is expressed in meters. When more than one family of particles are present, for example lithics and pumice, we will use the subscript $j$ to distinguish among them. Consequently, the function $f_{j}(D)$ will denote the number concentration of particles of the $j$ th family.

Given a particle size distribution $f_{j}(D)$, we observe that its "shape" can be quantified through the moments $M_{j}^{(i)}$ (Hazewinkel, 2001), defined by

$$
M_{j}^{(i)}=\int_{0}^{+\infty} D^{i} f_{j}(D) d D
$$

The particular definition of $f_{j}(D)$ we adopt, expressing the number concentration of particles of size $D$, allows for the following physical interpretation of the first four moments:

- $M_{j}^{(0)}$ is the total number of particles of the $j$ th family (per unit volume).

- $M_{j}^{(1)}$ is the sum of the particle's diameters of the $j$ th family (per unit volume).

- $M_{j}^{(2)}$ is the total surface area of particles of the $j$ th family (per unit volume).

- $\frac{\pi}{6} M_{j}^{(3)}$ is the total volume of particles of the $j$ th family (per unit volume) or the local volume fraction of the $j$ th dispersed phase, also denoted with $\alpha_{\mathrm{s}, j}$. The multiplying factor $\frac{\pi}{6}$ is obtained assuming spherical particles. For particles with different shape, if volume scales with the third power of length, we can still relate the particle volume $V$ with the particle length $D$ through a volumetric shape factor $k_{\mathrm{v}}$ such as $V=k_{\mathrm{v}} L^{3}$.

We also note that the central moments (i.e., those taken about the mean) can be expressed as function of the raw 
moments (i.e., those taken about zero as in Eq. 1), and in this way it is possible to relate the moments of the distribution with the mean, variance, skewness and kurtosis. Furthermore, a mean particle size can be defined as the ratio of the moments $M_{j}^{(i+1)} / M_{j}^{(i)}$ for any value of $i$. For example, the Sauter mean diameter (defined as the ratio between the mean volume and the mean surface area) is obtained by setting $i=2$, giving $L_{j, 32}=M_{j}^{(3)} / M_{j}^{(2)}$. Similarly, it is possible to define the mean particle length averaged with respect to particle number density $L_{j, 10}=M_{j}^{(1)} / M_{j}^{(0)}$, i.e., the sum of the lengths of particles (per unit volume) divided by the number of particles (per unit volume), and the mean particle length averaged with respect to particle volume fraction $L_{j, 43}=M_{j}^{(4)} / M_{j}^{(3)}$.

The motivation for the introduction of the moments is to minimize computational costs by avoiding the discretization of the size distribution in several classes, and nevertheless to capture the polydispersity of the flow through the correct description of the evolution of the moments (Carneiro, 2011). The moments approach also allows one to treat interparticle processes such as particle aggregation and fragmentation that strongly depend on and affect the GSD of the mixture (Marchisio et al., 2003). The moments and the corresponding transport velocities appear naturally in the mathematical formulation as a direct consequence of the integration of the Eulerian particle equations over the diameter spectrum, as will be shown in the next section.

\subsection{Moments of other quantities}

In the plume model, several quantities characteristic of the particles, such as settling velocity, density and specific heat capacity, are also defined as functions of the particle diameter, and thus we can define their moments as was carried out for the particle size distribution $f_{j}(D)$. In general, for a quantity $\psi_{j}$ that is a function of the diameter $D$, we define its moments as

$\psi_{j}^{(i)}=\frac{1}{M_{j}^{(i)}} \int_{0}^{+\infty} \psi_{j}(D) D^{i} f_{j}(D) d D$.

As a first example, we consider here the moments of particle density $\rho_{\mathrm{s}}$. In particular, following Bonadonna and Phillips (2003), density of lithics is assumed to be constant, whereas density of pumices $\rho_{\text {s,pum }}(D)$ with diameter $D<D_{2}$ (here equal to $2 \mathrm{~mm}$ ) is assumed to decrease and to reach the lithic density value when the fragment diameter decreases below $D_{1}$ (here equal to $8 \mu \mathrm{m}$ ). Substituting the expression for the particle density of the $j$ th particle family in Eq. (2), we obtain the moments of the density as

$\rho_{\mathrm{s}, j}^{(i)}=\frac{1}{M_{j}^{(i)}} \int_{0}^{+\infty} \rho_{\mathrm{s}, j}(D) D^{i} f_{j}(D) d D$.
We remark that moments of different order are generally different, they will only be equal $\left(\rho_{\mathrm{s}, j}^{(l)}=\rho_{\mathrm{s}, j}^{(m)}, l \neq m\right)$ in two limiting cases: for a monodisperse distribution with diameter $D^{*}$ and density $\rho_{\mathrm{s}}^{*}$, i.e., $f_{j}(D)=\delta\left(D-D^{*}\right)$ (where $\delta$ is the Dirac-delta function) and $\rho_{\mathrm{s}, j}\left(D^{*}\right)=\rho_{\mathrm{s}}^{*}$; or if all particles have the same density, i.e., $\rho_{\mathrm{s}, j}(D)=\rho_{\mathrm{s}, j}^{*}, \forall D$. In both cases, $\rho_{\mathrm{s}, j}^{(i)}=\rho_{\mathrm{s}, j}^{*}, \forall i$. Otherwise, there is no reason, e.g., for $\rho_{\mathrm{s}, j}^{(1)}$ and $\rho_{\mathrm{s}, j}^{(3)}$ to be the same, as they represent length- and volumeweighted density averages, respectively. For our application, we are interested mostly in the volumetric-averaged density $\rho_{\mathrm{s}, j}^{(3)}$, i.e., the average mass per unit volume of particles from now on denoted with $\widetilde{\rho}_{\mathrm{s}, j}$.

The moments defined by Eq. (3) can also be used to define other properties of the gas-particles mixture. For example, it follows from the definition of the moments that if we have a mixture of a gas with density $\rho_{\mathrm{g}}$ and a family of polydisperse distributions of particles with density $\rho_{\mathrm{s}, j}=\rho_{\mathrm{s}, j}(D)$, the mixture density is given by

$$
\begin{aligned}
\rho_{\text {mix }} & =\sum_{j} \alpha_{\mathrm{s}, j} \widetilde{\rho}_{\mathrm{s}, j}+\left(1-\sum_{j} \alpha_{\mathrm{s}, j}\right) \rho_{\mathrm{g}} \\
& =\sum_{j} \frac{\pi}{6} M_{j}^{(3)} \rho_{\mathrm{s}, j}^{(3)}+\left(1-\sum_{j} \frac{\pi}{6} M_{j}^{(3)}\right) \rho_{\mathrm{g}},
\end{aligned}
$$

and consequently the mass fraction of the $j$ th solid phase with respect to the gas-particles mixture is given by

$x_{\mathrm{s}, j}=\frac{\alpha_{\mathrm{s}, j} \widetilde{\rho}_{\mathrm{s}, j}}{\rho_{\mathrm{mix}}}=\frac{\frac{\pi}{6} M_{j}^{(3)} \rho_{\mathrm{s}, j}^{(3)}}{\sum_{j} \frac{\pi}{6} M_{j}^{(3)} \rho_{\mathrm{s}, j}^{(3)}+\left(1-\sum_{j} \frac{\pi}{6} M_{j}^{(3)}\right) \rho_{\mathrm{g}}}$.

We also remark here that the gas phase is a mixture of atmospheric air, entrained in the plume during the rise in the atmosphere, and a volcanic gas component, generally water vapor. In the following, we will use the subscript atm to denote the atmospheric air and $\mathrm{wV}$ for the volcanic water vapor.

In contrast to the approach used in Barsotti et al. (2008), where a constant settling velocity for each class is provided by the user, here several models have been implemented in the code (Pfeiffer et al., 2005; Textor et al., 2006a, b). For the application presented in this work, the settling velocity is defined as a function of the particle diameter and density as in Textor et al. (2006a):

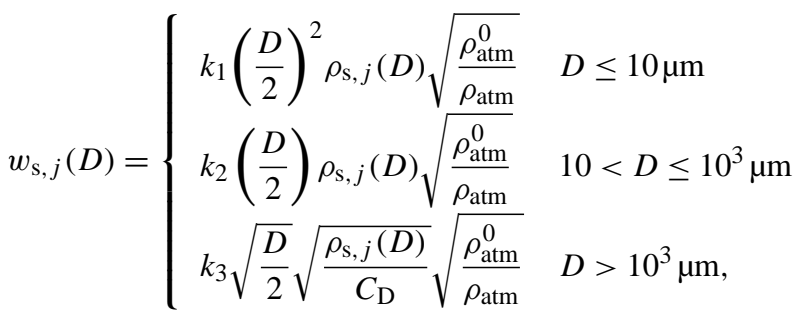

where $k_{1}=1.19 \times 10^{5} \mathrm{~m}^{2} \mathrm{~kg}^{-1} \mathrm{~s}^{-1}, k_{2}=8 \mathrm{~m}^{3} \mathrm{~kg}^{-1} \mathrm{~s}^{-1}$ and $k_{3}=4.833 \mathrm{~m}^{2} \mathrm{~kg}^{-1 / 2} \mathrm{~s}^{-1}$. The drag coefficient $C_{\mathrm{D}}$ is a parameter accounting for the particle surface roughness, and 
for this work we used a value of 0.75 as in Carey and Sparks (1986).

As carried out for particle density, it is possible to evaluate the moments $w_{\mathrm{s}, j}^{(i)}$ of the settling velocity $w_{\mathrm{s}, j}(D)$, defined as

$w_{\mathrm{s}, j}^{(i)}=\frac{1}{M_{j}^{(i)}} \int_{0}^{+\infty} w_{\mathrm{s}, j}(D) D^{i} f_{j}(D) d D$

and representing weighted integrals of the settling velocity over the size spectrum. Again, moments of different order are generally different. There is no reason, e.g., for $w_{\mathrm{s}, j}^{(2)}$ and $w_{\mathrm{s}, j}^{(3)}$ to be the same, as they represent surface and volumeweighted averages, respectively.

Finally, it is possible to define the moments $C_{\mathrm{s}, j}^{(i)}$ of the particles' specific heat capacity $C_{\mathrm{s}, j}$ :

$C_{\mathrm{s}, j}^{(i)}=\frac{1}{M_{j}^{(i)}} \int_{0}^{+\infty} C_{\mathrm{s}, j}(D) D^{i} f_{j}(D) d D$.

We observe that for the specific heat capacity, we are generally not interested in a volumetric average but in the mass average, denoted here with the notation $\bar{C}_{\mathrm{s}, j}$ and given by the following expression:

$$
\begin{aligned}
\bar{C}_{\mathrm{s}, j} & =\int_{0}^{+\infty} C_{\mathrm{s}, j}(D) \frac{\rho_{\mathrm{s}, j}(D) D^{3}}{\widetilde{\rho}_{\mathrm{s}, j} M_{j}^{(3)}} f_{j}(D) d D \\
& =\frac{1}{\widetilde{\rho}_{\mathrm{s}, j}}\left[C_{\mathrm{s}, j} \rho_{\mathrm{s}, j}\right]^{(3)} .
\end{aligned}
$$

\subsection{Mass fraction distribution}

While in chemical engineering, where the method of moments is commonly used, the particle number distribution $f_{j}(D)$ is generally preferred to describe the polydispersity of the particles; in volcanology it is more common to use a mass fraction distribution $\gamma_{j}(\phi)$, defined as a function of the Krumbein phi $(\phi)$ scale:

$\phi=-\log _{2} \frac{1000 D}{D_{0}}$,

where $D$ is the diameter of the particle expressed in meters, and $D_{0}$ is a reference diameter, equal to $1 \mathrm{~mm}$ (to make the equation dimensionally consistent).

In this case, the distribution $\gamma_{j}(\phi)$ represents the mass fraction of particles (mass per unit mass of the gas-particles mixture) of the $j$ th family with diameter between $\phi$ and $\phi+d \phi$. Again, the shape of the distribution $\gamma_{j}(\phi)$ can be characterized by its moments $\Pi_{j}^{i}$, defined by

$$
\Pi_{j}^{(i)}=\int_{-\infty}^{+\infty} \phi^{i} \gamma_{j}(\phi) d \phi .
$$

Also in this case the particular definition of $\gamma_{j}(\phi)$ allows for a physical interpretation of the moments; for example, the moment $\Pi_{j}^{(0)}$ is the mass fraction of the $j$ th solid phase $x_{\mathrm{s}, j}$ with respect to the gas-particles mixture. As carried out for particle number distribution, it is possible to define a mean particle size in terms of the moments of the mass fraction distribution as $\Pi_{j}^{(i+1)} / \Pi_{j}^{(i)}$; this ratio, for $i=0$, gives the massaveraged diameter, corresponding to the volume-averaged diameter $L_{j, 43}=M_{j}^{(4)} / M_{j}^{(3)}$ when the density $\rho_{\mathrm{s}, j}(\phi)$ is constant.

Again, it is possible to define the moments of other quantities $\psi_{j}(\phi)$ in terms of the continuous distribution of mass fraction $\gamma_{j}(\phi)$ as

$\psi_{j}^{(i)}=\frac{1}{\Pi_{j}^{(i)}} \int_{-\infty}^{+\infty} \psi_{j}(\phi) \phi^{i} \gamma_{j}(\phi) d \phi$.

For example, when the mass fraction distribution $\gamma_{j}(\phi)$ is used, the mass-averaged heat capacity $\bar{C}_{\mathrm{s}, j}$ is given by the following expression:

$\bar{C}_{\mathrm{s}, j}=\frac{1}{x_{\mathrm{s}, j}} \int_{-\infty}^{+\infty} C_{\mathrm{s}, j}(\phi) \gamma_{\mathrm{s}, j}(\phi) d \phi=C_{\mathrm{s}, j}^{(0)}$

and the volumetric-averaged density, i.e., the mass of particles per unit volume, can be evaluated from

$\frac{1}{\widetilde{\rho}_{\mathrm{s}, j}}=\frac{1}{x_{\mathrm{s}, j}} \int_{-\infty}^{+\infty} \frac{\gamma_{\mathrm{s}, j}(\phi)}{\rho_{\mathrm{s}, j}(\phi)} d \phi=\left[\frac{1}{\rho_{\mathrm{s}, j}}\right]^{(0)}$.

\section{Plume model}

In this section we describe the assumption and the equations of the model. As in Bursik (2001), the model assumes an homogeneous mixture of particles and gases with thermal and mechanical equilibrium between all phases. Aggregation and breakage effects are not considered and consequently density does not change with time. Finally, the model does not consider effects of humidity and water phase changes.

The equation set for the plume rise model is solved in a 3 -D coordinate system $(s, \eta, \theta)$ by considering the bulk properties of the eruptive mixture (see Fig. 1). The plume is assumed with a circular section in the plane normal to the centerline trajectory with curvilinear coordinate $s$, a top-hat profile of the velocity along the centerline, an inclination on the ground defined by an angle $\eta$ between the axial direction and the horizon, and an angle $\theta$ in the horizontal plane $(x, y)$ with respect to the $x$ axis. These angles are needed to describe the evolution of weak explosive eruptions that are strongly affected by atmospheric conditions.

Following Bursik et al. (1992) and Ernst et al. (1996), the conservation of flux of particles with size $D$ of the $j$ th family 


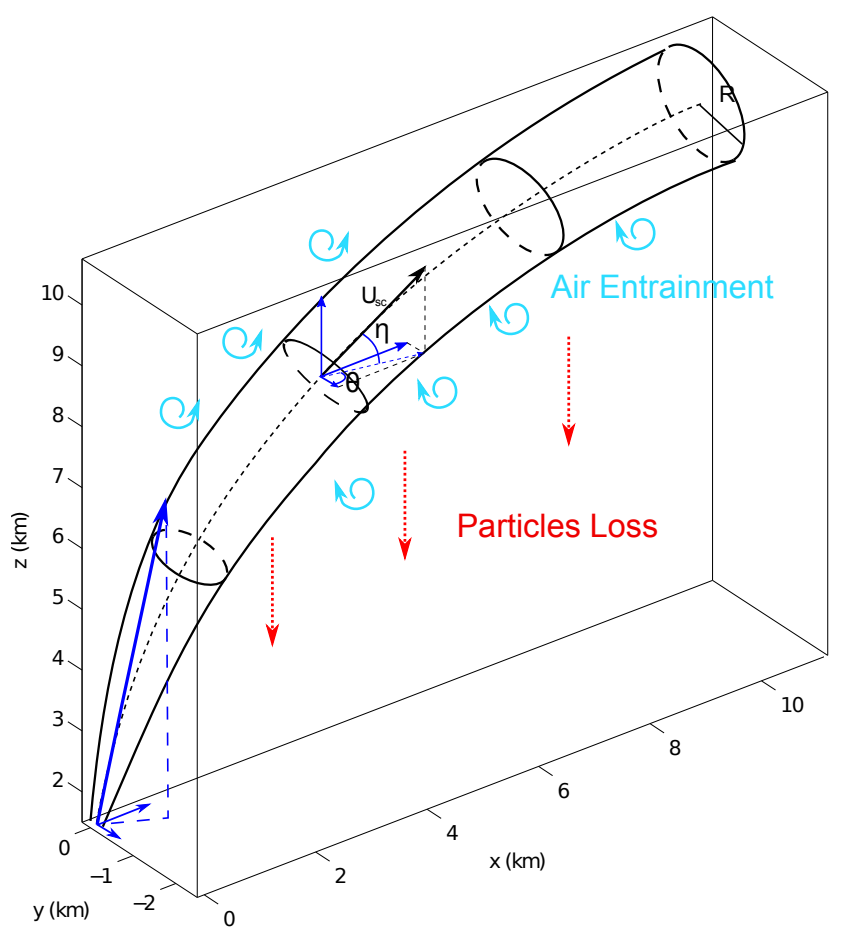

Figure 1. Schematic representation of the Eulerian plume model. The dashed black line represent the axis of the curvilinear coordinate $s$.

is given by

$\frac{d}{d s}\left(f_{j}(D) \pi r^{2} U_{\mathrm{sc}}\right)=-2 \pi r p w_{\mathrm{s}, j}(D), f_{j}(D)$

where $r$ is characteristic plume radius, $U_{\mathrm{sc}}$ represents the velocity of the plume cross section along its centerline (a tophat profile is assumed) and $p$ is the probability that an individual particle will fall out of the plume, defined as a function of an entrainment coefficient $\alpha$ as

$p=\frac{\left(1+\frac{6}{5} \alpha\right)^{2}-1}{\left(1+\frac{6}{5} \alpha\right)^{2}+1}$.

Equation (14) states that the number of particles of the $j$ th family with size $D$ lost from the plume is proportional to the number of particles at the plume margin, given by $f_{j}(D) \times$ $2 \pi r$, to the settling velocity $w_{\mathrm{s}, j}(D)$ and to the probability factor $p$.

Now, multiplying both the sides of Eq. (14) for $D^{i}$ and then integrating over the size spectrum $[0,+\infty]$, we obtain the following conservation equations for the moments $M_{j}^{(i)}$ :

$$
\frac{d}{d s}\left(M_{j}^{(i)} U_{\mathrm{sc}} r^{2}\right)=-2 r p w_{\mathrm{s}, j}^{(i)} M_{j}^{(i)} .
$$

If we compare our formulation with that presented in Barsotti et al. (2008), where the effects of a polydisperse solid phase are taken into account partitioning the size spectrum in a finite number $N$ of solid classes, the set of Eq. (16) replaces the $N$ mass conservation equations for the $N$ particulate classes.

From Eq. (14), if we multiply both the terms by the mass of the particles of size $D$, given by $\frac{\pi}{6} D^{3} \rho_{\mathrm{s}, j}(D)$, we obtain the additional equation

$$
\begin{aligned}
& \frac{d}{d s}\left(f_{j}(D) \frac{\pi}{6} D^{3} \rho_{\mathrm{s}, j}(D) \pi r^{2} U_{\mathrm{sc}}\right)= \\
& \quad-2 \pi r p w_{\mathrm{s}, j}(D) f_{j}(D) \frac{\pi}{6} D^{3} \rho_{\mathrm{s}, j}(D)
\end{aligned}
$$

and, integrating over the size spectrum,

$$
\frac{d}{d s}\left(U_{\mathrm{sc}} r^{2} \frac{\pi}{6} M_{j}^{(3)} \rho_{\mathrm{s}, j}^{(3)}\right)=-2 r p \frac{\pi}{6} M^{(3)}\left[w_{\mathrm{s}, j} \rho_{\mathrm{s}, j}\right]^{(3)},
$$

where on the left-hand side the term $\frac{\pi}{6} M_{j}^{(3)} \rho_{\mathrm{s}, j}^{(3)}$ represents the volume average bulk density of the particles of the $j$ th family (i.e., the mass of particles of the $j$ th family per unit volume of gas-particles mixture, denoted with the superscript $B, \rho_{\mathrm{s}, j}^{B}$ ), while on the right-hand side the term $\left[w_{\mathrm{s}, j} \rho_{\mathrm{s}, j}\right]^{(3)}$ represents the mass-averaged settling velocity of the particles of the $j$ th family multiplied by the volume-averaged particle density. Equation (18) is the mass conservation equation for the $j$ th family of particles, relating the variation of the mass flux of particles within the plume with the loss at the plume margin.

Now, following the same procedure, we reformulate the other conservation equations describing the steady-state ascent of the plume in terms of the moments of the continuous distributions of sizes, densities and settling velocities instead of the averages over a finite number of classes of particles with different size.

First of all, we derive the conservation equation for the mixture mass. As in the plume theory, we assume that the entrainment, due to both turbulence in the rising buoyant jet and to the crosswind field, is parameterized through the use of two entrainment coefficients, $\alpha_{\epsilon}$ and $\gamma_{\epsilon}$. The theory assumes that the efficiency of mixing with ambient air is proportional to the product of a reference velocity (the vertical plume velocity in one case and the wind field component along the plume centerline in the other), by $\alpha_{\epsilon}$ and $\gamma_{\epsilon}$ (Morton, 1959; Briggs, 1975; Wright, 1984; Weil, 1988). Thus, following Hewett et al. (1971) and Bursik (2001), we define the entrainment velocity $U_{\epsilon}$ as a function of wind speed, $U_{\text {atm }}$, as well as axial plume speed, $U_{\mathrm{sc}}$ :

$U_{\epsilon}=\alpha_{\epsilon}\left|U_{\mathrm{sc}}-U_{\mathrm{atm}} \cos \phi\right|+\gamma_{\epsilon}\left|U_{\mathrm{atm}} \sin \phi\right|$,

where $\alpha_{\epsilon}\left|U_{\mathrm{sc}}-U_{\mathrm{atm}} \cos \phi\right|$ is entrainment by radial inflow minus the amount swept tangentially along the plume margin by the wind, and $\gamma_{\epsilon}\left|U_{\mathrm{atm}} \sin \phi\right|$ is entrainment from wind. With this notation, the total mass conservation equa- 
tion solved by the model becomes

$$
\begin{aligned}
\frac{d}{d s}\left(\rho_{\mathrm{mix}} U_{\mathrm{sc}} r^{2}\right) & =2 r \rho_{\mathrm{atm}} U_{\epsilon} \\
& -2 r p \sum_{j} \frac{\pi}{6} M_{j}^{(3)}\left[w_{\mathrm{s}, j} \rho_{\mathrm{s}, j}\right]^{(3)},
\end{aligned}
$$

stating that the variation of mass flux (left-hand-side term) is due to air entrainment (first right-hand-side term) and loss of solid particles (second right-hand-side term), as obtained from Eq. (18).

From Newton's second law and the variation of mass flux, we can derive also the horizontal and vertical components of the momentum balance solved by the model as

$$
\begin{aligned}
& \frac{d}{d s}\left(\rho_{\mathrm{mix}} U_{\mathrm{sc}} r^{2}\left(u-U_{\mathrm{atm}}\right)\right)= \\
& \quad-r^{2} \rho_{\text {mix }} w \frac{d U_{\mathrm{atm}}}{d z}-2 u p r \sum_{j} \frac{\pi}{6} M_{j}^{(3)}\left[w_{\mathrm{s}, j} \rho_{\mathrm{s}, j}\right]^{(3)},
\end{aligned}
$$

and

$$
\begin{aligned}
& \frac{d}{d s}\left(\rho_{\text {mix }} U_{\mathrm{sc}} r^{2} w\right)= \\
& \quad g r^{2}\left(\rho_{\mathrm{atm}}-\rho_{\text {mix }}\right)-2 w p r \sum_{j} \frac{\pi}{6} M_{j}^{(3)}\left[w_{\mathrm{s}, j} \rho_{\mathrm{s}, j}\right]^{(3)},
\end{aligned}
$$

where the two components of plume velocity along the horizontal and vertical axes are $u$ and $w$, respectively, and they are linked by the relation $U_{\mathrm{sc}}=\sqrt{u^{2}+w^{2}}$. In the right-hand side of Eq. (21) the terms related to the exchange of momentum due to the wind (Barsotti et al., 2008) and to momentum loss from the fall of solid particles appear. Similar contributions are evident in the right-hand-side term of Eq. (22) where the vertical momentum is changed by the gravitational acceleration term and the fall-out of particles.

Now, following the notation adopted above and denoting with $T$ the mixture temperature, the equation for conservation of thermal energy solved by the model is written as

$$
\begin{aligned}
& \frac{d}{d s}\left(\rho_{\mathrm{mix}} U_{\mathrm{sc}} r^{2} C_{\mathrm{mix}} T\right)=2 r \rho_{\mathrm{atm}} U_{\epsilon} C_{\mathrm{atm}} T_{\mathrm{atm}} \\
& \quad-r^{2} w \rho_{\mathrm{atm}} g-2 T p r \sum_{j} \frac{\pi}{6} M_{j}^{(3)}\left[C_{\mathrm{s}, j} w_{\mathrm{s}, j} \rho_{\mathrm{s}, j}\right]^{(3)} .
\end{aligned}
$$

The first term on right-hand side describes the cooling of the plume due to ambient air entrainment, the second one takes into account atmospheric thermal stratification, and the third term allows for heat loss due to loss of solid particles. Again, this last term is obtained writing the heat loss for the particles of size $D$, and then integrating over the size spectrum. A thermal equilibrium between solid and gaseous phases is assumed. In Eq. (23) $C_{\text {atm }}$ and $C_{\text {mix }}$ are the heat capacity of the entrained atmospheric air and of the mixture, respectively, the latter being defined as

$C_{\text {mix }}=\left(1-\sum_{j} x_{\mathrm{s}, j}\right) C_{p, g}+\sum_{j} x_{\mathrm{s}, j} \bar{C}_{\mathrm{s}, j}$ or, in terms of the bulk densities $\rho_{\mathrm{atm}}^{B}=x_{\mathrm{atm}} \rho_{\mathrm{mix}}, \rho_{\mathrm{wv}}^{B}=$ $x_{\mathrm{wv}} \rho_{\mathrm{mix}}$ and $\rho_{\mathrm{s}, j}^{B}=\frac{\pi}{6} M_{j}^{(3)} \widetilde{\rho}_{\mathrm{s}, j}$, as

$C_{\mathrm{mix}}=\frac{\rho_{\mathrm{atm}}^{B} C_{\mathrm{atm}}+\rho_{\mathrm{wv}}^{B} C_{\mathrm{wv}}+\sum_{j} \rho_{\mathrm{s}, j}^{B} \bar{C}_{\mathrm{s}, j}}{\rho_{\mathrm{atm}}^{B}+\rho_{\mathrm{wv}}^{B}+\sum_{j} \rho_{\mathrm{s}, j}^{B}}$.

From this expression, if we multiply all the terms at the numerator and the denominator of the right-hand side by $U_{\mathrm{sc}} r^{2}$ and we differentiate with respect to $s$, we obtain after some cancellation and algebra manipulations the following equation for the variation of the mixture specific heat with $s$ :

$$
\begin{aligned}
\frac{d C_{\mathrm{mix}}}{d s} & =\frac{1}{\rho_{\mathrm{mix}} U_{\mathrm{sc}} r^{2}}\left[\left(C_{\mathrm{atm}}-C_{\mathrm{mix}}\right) \frac{d}{d s}\left(\rho_{\mathrm{atm}}^{B} U_{\mathrm{sc}} r^{2}\right)\right. \\
& \left.+\sum_{j}\left(\bar{C}_{\mathrm{s}, j}-C_{\mathrm{mix}}\right) \frac{d}{d s}\left(\rho_{\mathrm{s}, j}^{B} U_{\mathrm{sc}} r^{2}\right)\right] \\
& +\sum_{j} \frac{\rho_{\mathrm{s}, j}^{B}}{\rho_{\mathrm{mix}}}\left[\frac{\frac{d}{d s}\left(\bar{C}_{\mathrm{s}, j} \rho_{\mathrm{s}, j}^{B} U_{\mathrm{sc}} r^{2}\right)}{\rho_{\mathrm{s}, j}^{B} U_{\mathrm{sc}} r^{2}}\right. \\
& \left.-\frac{\bar{C}_{\mathrm{s}, j} \frac{d}{d s}\left(\rho_{\mathrm{s}, j}^{B} U_{\mathrm{sc}} r^{2}\right)}{\rho_{\mathrm{s}, j}^{B} U_{\mathrm{sc}} r^{2}}\right] .
\end{aligned}
$$

Now, substituting the expressions for the derivatives appearing in each term on the right-hand side, we obtain the equation for the variation rate of mixture specific heat in terms of the moments:

$$
\begin{aligned}
\frac{d C_{\mathrm{mix}}}{d s} & =\frac{1}{\rho_{\operatorname{mix}} U_{\mathrm{sc}} r^{2}}\left[C_{\mathrm{atm}} 2 r \rho_{\mathrm{atm}} U_{\epsilon}-C_{\mathrm{mix}}\left(2 r \rho_{\mathrm{atm}} U_{\epsilon}\right.\right. \\
& \left.-2 p r \sum_{j} \frac{\pi}{6} M_{j}^{(3)}\left[w_{\mathrm{s}, j} \rho_{\mathrm{s}, j}\right]^{(3)}\right) \\
& \left.-2 p r \sum_{j} \frac{\pi}{6} M_{j}^{(3)}\left[w_{\mathrm{s}, j} \rho_{\mathrm{s}, j} C_{\mathrm{s}, j}\right]^{(3)}\right] .
\end{aligned}
$$

Similarly, a gas constant $R_{\mathrm{g}}$ can be defined as a weighted average of the gas constant for the entrained atmospheric air $R_{\mathrm{atm}}$ and the gas constant of the volcanic water vapor $R_{\mathrm{wv}}$

$R_{\mathrm{g}}=\frac{\rho_{\mathrm{atm}}^{B} R_{\mathrm{atm}}+\rho_{\mathrm{wv}}^{B} R_{\mathrm{wv}}}{\rho_{\mathrm{atm}}^{B}+\rho_{\mathrm{wv}}^{B}}$,

and a conservation equation can be derived, knowing that the variation of gaseous mass fraction with height is solely due to entrained air:

$\frac{d R_{\mathrm{g}}}{d s}=\frac{R_{\mathrm{atm}}-R_{\mathrm{g}}}{\rho_{\mathrm{mix}}\left(1-x_{\mathrm{s}}\right) U_{\mathrm{sc}} r^{2}} \times 2 r \rho_{\mathrm{atm}} U_{\epsilon}$.

This formulation reduces, for particular cases, to the expressions of Woods (1988) and Glaze and Baloga (1996). Equations (27) and (29) are needed in order to close the system of 
equations and recover the new values of the temperature and the mixture density once the system of ordinary differential equations is integrated. Otherwise, without the solutions of Eqs. (27) and (29), we should use the old values of $\rho_{\text {mix }}$ and $C_{\text {mix }}$ at $s$ to obtain the values of the temperature at $s+d s$ from the lumped term $\left(\rho_{\text {mix }} U_{\mathrm{sc}} r^{2} C_{\text {mix }} T\right)$ obtained integrating Eq. (23).

Finally, as in Bursik (2001), the equations expressing the coordinate transformation between $(x, y, z)$ and $(s, \eta, \theta)$ are given by

$$
\frac{d z}{d s}=\sin \eta, \quad \frac{d x}{d s}=\cos \eta \cos \theta, \quad \frac{d y}{d s}=\cos \eta \sin \theta .
$$

\subsection{Mass fraction distribution}

Similarly to the distribution of particle number $f_{j}(D)$ and the moments $M_{j}^{(i)}$, it is possible to derive a set of conservation equations in terms of the moments $\Pi_{j}^{(i)}$ of the mass fraction distribution $\gamma_{j}(\phi)$ expressed as a function of the Krumbein scale.

In this case, the conservation of mass flux of particles with size $\phi$ of the $j$ th family is written as

$$
\frac{d}{d s}\left(\rho_{\text {mix }} \gamma_{j}(\phi) \pi r^{2} U_{\mathrm{sc}}\right)=-2 \pi r p w_{\mathrm{s}, j}(\phi) \rho_{\mathrm{mix}} \gamma_{j}(\phi) .
$$

Multiplying both sides of the equation by $\phi^{i}$ and integrating over the size spectrum $[-\infty,+\infty]$, we obtain the following conservation equations for the moments of the continuous distributions $\gamma_{j}(\phi)$ :

$\frac{d}{d s}\left(\Pi_{j}^{(i)} \rho_{\text {mix }} U_{\mathrm{sc}} r^{2}\right)=-2 r p \rho_{\operatorname{mix}} w_{\mathrm{s}, j}^{(i)} \Pi_{j}^{(i)}$.

For $i=0$, the equations of conservation of the moments give

$$
\frac{d}{d s}\left(x_{\mathrm{s}, j} \rho_{\mathrm{mix}} U_{\mathrm{sc}} r^{2}\right)=-2 r p \rho_{\mathrm{mix}} w_{\mathrm{s}, j}^{(0)} x_{\mathrm{s}, j}
$$

expressing the loss of mass flux of the particles of the $j$ th family and thus we can write the total mass conservation equation as

$$
\frac{d}{d s}\left(\rho_{\text {mix }} U_{\mathrm{sc}} r^{2}\right)=2 r \rho_{\mathrm{atm}} U_{\epsilon}-2 r p \rho_{\text {mix }} \sum_{j} w_{\mathrm{s}, j}^{(0)} \Pi_{j}^{(0)} .
$$

From the variation of mass flux, as was carried out for the distribution of particle number $f_{j}(D)$ and the moments $M_{j}^{(i)}$, we derive the horizontal and vertical components of the mo- mentum balance:

$$
\begin{aligned}
& \frac{d}{d s}\left(\rho_{\text {mix }} U_{\mathrm{sc}} r^{2}\left(u-U_{\mathrm{atm}}\right)\right)= \\
& \quad-r^{2} \rho_{\text {mix }} w \frac{d U_{\mathrm{atm}}}{d z}-2 u p r \rho_{\operatorname{mix}} \sum_{j} w_{\mathrm{s}, j}^{(0)} \Pi_{j}^{(0)},
\end{aligned}
$$

$$
\begin{aligned}
& \frac{d}{d s}\left(\rho_{\text {mix }} U_{\mathrm{sc}} r^{2} w\right)= \\
& \quad g r^{2}\left(\rho_{\text {atm }}-\rho_{\text {mix }}\right)-2 w \operatorname{pr} \rho_{\text {mix }} \sum_{j} w_{\mathrm{s}, j}^{(0)} \Pi_{j}^{(0)} .
\end{aligned}
$$

The equation for conservation of thermal energy is

$$
\begin{aligned}
& \frac{d}{d s}\left(\rho_{\text {mix }} U_{\mathrm{sc}} r^{2} C_{\mathrm{mix}} T\right)=2 r \rho_{\mathrm{atm}} U_{\epsilon} C_{\mathrm{atm}} T_{\mathrm{atm}} \\
& \quad-r^{2} w \rho_{\mathrm{atm}} g-2 T \operatorname{pr} \rho_{\text {mix }} \sum_{j}\left[C_{\mathrm{s}, j} w_{\mathrm{s}, j}\right]^{(0)} \Pi_{j}^{(0)}
\end{aligned}
$$

and the equation for the variation rate of mixture specific heat in terms of the moments of the mass fraction distribution is written as

$$
\begin{aligned}
\frac{d C_{\mathrm{mix}}}{d s} & =\frac{1}{\rho_{\mathrm{mix}} U_{\mathrm{sc}} r^{2}}\left[C_{\mathrm{atm}} 2 r \rho_{\mathrm{atm}} U_{\epsilon}-C_{\mathrm{mix}}\left(2 r \rho_{\mathrm{atm}} U_{\epsilon}\right.\right. \\
& \left.-2 r p \rho_{\operatorname{mix}} \sum_{j} w_{\mathrm{s}, j}^{(0)} \Pi_{j}^{(0)}\right) \\
& \left.-2 \operatorname{pr} \rho_{\operatorname{mix}} \sum_{j}\left[C_{\mathrm{s}, j} w_{\mathrm{s}, j}\right]^{(0)} \Pi_{j}^{(0)}\right] .
\end{aligned}
$$

The formulation of the equations for the gas constant $R_{\mathrm{g}}$ and the coordinates of the $(x, y, z)$ remain unchanged.

\section{Numerical scheme}

The plume rise equations are solved with a predictorcorrector Heun's scheme (Petzold and Ascher, 1998) that guarantees a second-order accuracy, keeping the execution time on the order of seconds. If we rewrite the system of ordinary differential equations with the following compact notation:

$\frac{d \boldsymbol{y}}{d s}=f(s, \boldsymbol{y}), \quad \boldsymbol{y}\left(s_{0}\right)=\boldsymbol{y}_{0}$,

where $y$ is the vector of the quantities in the left-hand sides of the conservation equations presented in the previous section, then the procedure for calculating the numerical solution by way of Heun's method (Süli and Mayers, 2003) is to first calculate the intermediate values $\tilde{\boldsymbol{y}}_{i+1}$ and then the solution $\boldsymbol{y}_{i+1}$ at the next integration point

$\tilde{\boldsymbol{y}}_{i+1}=\boldsymbol{y}_{i}+d s f\left(s_{i}, \boldsymbol{y}_{i}\right)$, predictor step

$\boldsymbol{y}_{i+1}=\boldsymbol{y}_{i}+\frac{d s}{2}\left(f\left(s_{i}, \boldsymbol{y}_{i}\right)+f\left(s_{i+1}, \tilde{\boldsymbol{y}}_{i+1}\right)\right)$, corrector step. 


\subsection{Quadrature method of moments}

We observe that to calculate the right-hand side for both the predictor and corrector step we need not only the moments $M^{(i)}$ but also the additional moments $\left[w_{\mathrm{s}}\right]^{i},\left[w_{\mathrm{s}} \rho_{\mathrm{s}}\right]^{(i)}$ and $\left[w_{\mathrm{s}} \rho_{\mathrm{s}} C_{\mathrm{s}}\right]^{(i)}$. As in Marchisio and Fox (2013), the integral in the definition of these moments is replaced by a quadrature formula and the moments, for a generic variable $\psi=\psi(D)$, are approximated as

$\psi^{(i)}=\frac{1}{M^{(i)}} \int_{0}^{+\infty} \psi(D) f(D) D^{i} d D \approx \sum_{l=1}^{N} \psi\left(D_{l}\right) D_{l}^{i} \omega_{l}$.

Here $\omega_{l}$ and $D_{l}$ are known as "weights" and "nodes" (or "abscissae") of the quadrature, respectively, and the accuracy of a quadrature formula is quantified by its degree. The degree of accuracy is equal to $d$ if the interpolation formula is exact when the integrand is a polynomial of an order less than or equal to $d$ and there exists at least one polynomial of an order $d+1$ that makes the interpolation formula inexact. In particular, an $N$ point Gaussian quadrature rule, is a quadrature rule constructed to yield an exact result for polynomials of degree $2 N-1$ or less by a suitable choice of the nodes $D_{l}$ and weights $\omega_{l}$ for $l=1, \ldots, N$ (Golub and Welsch, 1969).

The Wheeler algorithm, as presented in Marchisio and Fox (2013), provides an efficient $O\left(N^{2}\right)$ algorithm for finding a full set of weights and abscissas for a realizable moment set. The resulting nodes $D_{l}$ are always within the support (and therefore represent realizable values of the particle size), and the weights $\omega_{l}$ are always positive, ensuring that, when the quadrature is used, accurate results are obtained (Marchisio and Fox, 2013). Nevertheless, these properties are respected only if the moment set is realizable, meaning that there exists a particle size distribution resulting in that specific set of moments.

A strategy that might overcome the problem of moment corruption (i.e., the transformation during the integration of the moment-transport equations of a realizable set of moments into an unrealizable one) is replacing unrealizable moment sets as soon as they appear. An algorithm of this kind was developed by McGraw (McGraw, 2006). The algorithm first verifies whether the moment set is realizable (by looking at the second-order difference vector or by looking at the Hankel-Hadamard determinants; Gautschi, 2004). If the moment set is unrealizable it proceeds with the correction. In the numerical model presented here, the implementation of the correction algorithm of Wright (1984) is derived from the version presented in Marchisio and Fox (2013).

Thus, in both the predictor and corrector step, the following algorithm is used:

- The nodes $D_{j, l}$ and weights $\omega_{j, l}$ are calculated with the Wheeler algorithm for $l=1, \ldots, N$.

- The quadrature formula (Eq. 41) is used to evaluate the moments $\left[w_{\mathrm{s}}\right]_{j}^{(i)},\left[w_{\mathrm{s}} \rho_{\mathrm{s}}\right]_{j}^{(i)}$ and $\left[w_{\mathrm{s}} \rho_{\mathrm{s}} C_{\mathrm{s}}\right]_{j}^{(i)}$.
- The right-hand side of the ODE's system (Eq. 39) is evaluated explicitly.

- The solution is advanced with the predictor (or the corrector) step of the Heun's scheme.

- For each particle family $j$, the moments $M_{j}^{(i)}(i=$ $0, \ldots, 2 N-1)$, if required, are corrected with the McGraw (or Wright) algorithm.

We observe that if the $j$ th family of particles is monodisperse with diameter $\bar{d}_{j}$, the Wheeler algorithm fed with the first two moments only gives a result of a single quadrature node $D_{j, 1}=\bar{d}_{j}$ with weight $\omega_{j, 1}=1$. This allows us also to use the model for the simplified case where the solid particle distribution is partitioned in a finite number of classes with constant size, assigning to each class a monodisperse distribution.

\subsection{Initial condition}

Initial conditions at the vent include the initial plume radius $\left(r_{0}\right)$, mixture velocity $\left(U_{\mathrm{sc}, 0}\right)$ and temperature $\left(T_{0}\right)$, gas mass fraction $\left(n_{0}\right)$ and the particle size distribution through the initial moments $M_{0}^{(i)}$. In the next section we derive analytically the moments of a specific initial distribution (a normal distribution in the Krumbein scale) for both the formulations based on the number of particles as a function of the particle diameter expressed in meters and the formulation based on the mass concentration expressed as a function of the phi scale.

\section{Lognormal distribution}

For the application presented in this work, the initial distribution $f(D)$ at the base of the plume is defined as a function of the particle diameter expressed in meters $(m)$, in order to give a corresponding normal distribution with parameters $\mu$ and $\sigma$ for the mass concentration expressed as a function of the Krumbein phi $(\phi)$ scale (when all the particles have the same density):

$\gamma(\phi)=\frac{K_{0}}{\sigma \sqrt{2 \pi}} e^{-\frac{(\phi-\mu)^{2}}{2 \sigma^{2}}}$,

where $K_{0}$ is a parameter that has to be chosen in order to satisfy the initial condition on the solid mass fraction.

Given the parameters $\mu$ and $\sigma$, the initial distribution $f(D)$ is then written as

$f(D)=\frac{6 C_{0}}{(-\sigma \ln 2) D^{4} \sqrt{2 \pi^{3}}} e^{-\frac{[-\ln (1000 D)-\mu \ln 2]^{2}}{2(\sigma \ln 2)^{2}}}$,

where $C_{0}$, analogously to $K_{0}$, is a parameter that has to be fixed in order to satisfy the initial condition prescribed for the mass (or volume) fraction of particles. 
We observe that if we introduce the following re-scaled variables for the diameter, the mean and the variance:

$\bar{D}=1000 D, \quad \bar{\mu}=-\mu \ln 2, \quad \bar{\sigma}=-\sigma \ln 2$,

then it is possible to rewrite the particle distribution $f(D)$ in terms of a lognormal distribution in the variable $\bar{D}$ with parameters $\bar{\mu}$ and $\bar{\sigma}$ :

$$
\begin{aligned}
\bar{f}(\bar{D}) & =\frac{6 \times 10^{12} C_{0}}{\pi \bar{D}^{3}} \frac{1}{\bar{\sigma} \bar{D} \sqrt{2 \pi}} e^{-\frac{[\ln (\bar{D})-\bar{\mu}]^{2}}{2 \bar{\sigma}^{2}}} \\
& =\frac{6 \times 10^{12} C_{0}}{\pi \bar{D}^{3}} \operatorname{lognorm}(\bar{D}, \bar{\mu}, \bar{\sigma}) .
\end{aligned}
$$

Consequently, we can evaluate the moments $M^{(i)}$ of $f(D)$ analytically from the moments of the lognormal distribution as

$$
M^{(i)}=\frac{6 C_{0}}{\pi} 10^{3(3-i)} \exp \left[(i-3) \bar{\mu}+\frac{1}{2}(i-3)^{2} \bar{\sigma}^{2}\right],
$$

and we obtain, for the third moment,

$M^{(3)}=\frac{6 C_{0}}{\pi} \Rightarrow C_{0}=\alpha_{\mathrm{s}}^{0}$,

where $\alpha_{\mathrm{s}}^{0}$ is the initial volume fraction of the particles in the solid-gas mixture.

From the expressions of the moments it follows also that, if the mass concentration expressed as a function of the Krumbein scale has a normal distribution, the Sauter mean diameter $D_{A}$ expressed in meters can be evaluated as

$D_{A}=L_{32}=\frac{M^{(3)}}{M^{(2)}}=10^{-3} \exp \left(\bar{\mu}-\frac{1}{2} \bar{\sigma}^{2}\right)$,

or, if expressed in $\phi$, as

$D_{A}^{\phi}=L_{32}^{\phi}=\mu+\frac{1}{2} \sigma^{2} \ln (2)$.

Processes involving the mutual interaction between particles and the interaction between the particles and the carrier fluid (friction and cohesion between the particles; viscous drag; chemical reactions between fluid and solid components) operate at the surface of the particles. For this reason the Sauter mean diameter, based on the specific area of the particles, is a convenient descriptor and it is important to remark that it differs from the mean $\mu$ of the lognormal distribution by a factor proportional to the variance $\sigma^{2}$. For numerical models describing the multi-phase (particulate) nature of the matter and which approximate the particle size distribution with an average size, it is hence more appropriate to use, as particle size representative of a lognormal distribution, the Sauter mean diameter than the mean diameter $\mu$. The difference between the two approximations is smaller the narrower the particle size distribution. We must also remark that, for particles in the inertial-dominated regime (e.g., $R e_{\mathrm{p}}>2000$ ), Loth et al. (2004) showed that the Sauter mean diameter is the effective diameter, regardless of particle shape, particle size distribution, particle density distribution or net volume fraction; for particles in the creeping flow regime $\left(R e_{\mathrm{p}} \ll 1\right)$ the effective mean diameter is the volume-width diameter.

When the Sauter mean diameter is used, also the variance and the standard deviation SD should be based on the specific surface area (Rietema, 1991). Hence,

$\sigma_{A}^{2}=\int_{0}^{+\infty}\left(\frac{1}{D}-\frac{1}{D_{A}}\right)^{2} \frac{\pi}{6} D^{3} f(D) d D$,

or expressed as a function of the moments:

$\sigma_{A}^{2}=\frac{M^{(1)} M^{(3)}-\left(M^{(2)}\right)^{2}}{\left(M^{(3)}\right)^{2}}$.

Finally, we note that if the particle density is constant and the mass concentration expressed as a function of the Krumbein scale has a lognormal distribution and both the Sauter mean diameter $L_{32}=M^{(3)} / M^{(2)}$ and the mean particle length averaged with respect to particle number density $L_{10}=M^{(1)} / M^{(0)}$ (or if the first 4 moments) are known, then we can solve for the re-scaled mean and variance $\bar{\mu}$ and $\bar{\sigma}$ the following system:

$$
\left\{\begin{array}{l}
L_{10}=10^{-3} \exp \left(\bar{\mu}-\frac{5}{2} \bar{\sigma}^{2}\right) \\
L_{32}=10^{-3} \exp \left(\bar{\mu}-\frac{1}{2} \bar{\sigma}^{2}\right)
\end{array} .\right.
$$

Once the re-scaled mean and variance are known, we can obtain $\mu$ and $\sigma$ in the Krumbein $\phi$ scale.

When the initial distribution is expressed for the mass fractions instead of the particle number, and the mass fraction written as a function of the Krumbein scale has a normal distribution with mean $\mu$ and variance $\sigma^{2}$, then the continuous distribution is given by Eq. (42). We observe that this expression of the distribution is not based on the assumption of constant density for the particles of different size.

In this case, the moments $\Pi^{(i)}$ are given by the following expression

$$
\Pi^{(i)}=K_{0} \sum_{j=0}^{\lceil i / 2\rceil}\left(\begin{array}{c}
i \\
2 j
\end{array}\right)(2 j-1) ! ! \sigma^{2 j} \mu^{i-2 j} .
$$

where the symbols \lceil\rceil and !! denote the integer part and the double factorial $\left(n ! !=\prod_{k=0}^{m}(n-2 k)\right.$, where $\left.m=\lceil n / 2\rceil-1\right)$, respectively.

Now, as the 0th moment is equal to the mass fraction of particles, we obtain $K_{0}=x_{\mathrm{s}}$. Furthermore, we observe that the mass fraction-averaged diameter in the $\phi$ scale is given by the ratio $\Pi^{(1)} / \Pi^{(0)}$, while the variance of the mass fraction distribution can be evaluated as 
Table 1. Input parameters used for the numerical simulations. Vent height is the elevation of the base of the column above sea level. The values $\rho_{1,2}$ and $D_{1,2}$ are used to compute the density of the particles as a function of the diameter, according to the formulation of Bonadonna and Phillips (2003). The values reported for $\mu$ and $\sigma$ define the range used for the uncertainty quantification and sensitivity analysis.

\begin{tabular}{llrrr}
\hline Parameters & Units & Test case 1 & Test case 2 & Test case 3 \\
\hline Vent radius & $\mathrm{m}$ & 27 & 27 & 708 \\
Vent velocity & $\mathrm{m} \mathrm{s}^{-1}$ & 135 & 135 & 275 \\
Vent temperature & $\mathrm{K}$ & 1273 & 1273 & 1053 \\
Vent gas mass fraction & & 0.03 & 0.03 & 0.05 \\
Vent height & $\mathrm{m}$ & 1500 & 1500 & 1500 \\
$\rho_{1}$ & $\mathrm{~kg} \mathrm{~m}^{-3}$ & 2000 & 2000 & 2000 \\
$\rho_{2}$ & $\mathrm{~kg} \mathrm{~m}^{-3}$ & 2600 & 2600 & 2600 \\
$D_{1}$ & $\mathrm{~m}$ & $8 \times 10^{-6}$ & $8 \times 10^{-6}$ & $8 \times 10^{-6}$ \\
$D_{2}$ & $\mathrm{~m}$ & $2 \times 10^{-3}$ & $2 \times 10^{-3}$ & $2 \times 10^{-3}$ \\
$\mu$ & $\phi$ & {$[-1.0 ; 3.0]$} & {$[-1.0 ; 3.0]$} & {$[-1.0 ; 3.0]$} \\
$\sigma$ & $\phi$ & {$[0.5 ; 2.5]$} & {$[0.5 ; 2.5]$} & {$[0.5 ; 2.5]$} \\
\hline
\end{tabular}

$\left[\Pi^{(2)} \Pi^{(0)}-\left(\Pi^{(1)}\right)^{2}\right] /\left(\Pi^{(0)}\right)^{2}$. These two quantities correspond to the parameters $\left(\mu, \sigma^{2}\right)$ generally used to describe the mass fraction when a normal distribution in the $\phi$ scale is assumed. For this reason, when we want to track the changes of the mass fraction-averaged diameter and its standard deviation (or variance) in the $\phi$ scale during the plume rise, it is preferred to use a formulation based on the moments $\Pi^{(i)}$ than the moments $M^{(i)}$.

\section{Application}

\subsection{Simulation inputs}

We applied the model to three different test cases with different vent and atmospheric conditions:

- test case 1 - low-flux plume without wind;

- test case 2 - low-flux plume with wind (weak bent plume);

- test case 3 - high-flux plume (strong plume).

The parameters used for the different test cases are listed in Table 1, while the atmospheric conditions are plotted in Fig. 3. For the low-flux plumes, a mass flow rate of $1.5 \times$ $10^{6} \mathrm{~kg} \mathrm{~s}^{-1}$ has been fixed, while for the strong plume the value is $1.5 \times 10^{9} \mathrm{~kg} \mathrm{~s}^{-1}$. The temperature pressure and density profiles used for the test case without wind (test case 1) are those defined by the International Organization for Standardization for the International Standard Atmosphere (Champion et al., 1985), while the profiles for the other two test cases come from reanalysis data.

For all the runs presented here, a single family of particles has been used, with a normal distribution (with parameters $\mu$ and $\sigma$ ) for the mass concentration as a function of the diameter expressed in the $\phi$ scale and with density varying with the particle diameter.
We first present a comparison of the plume profiles obtained with the three different descriptions presented in the previous sections and highlighted in the three colored boxes of Fig. 2 for the test case 2: method of moments for the particle number that is the function of the size expressed in meters; method of moments for the particle mass fraction that is the function of the size expressed in the $\phi$ scale; discretization in uniform bins in the $\phi$ scale. For this comparison, the mass flow rate at the vent is $1.5 \times 10^{6} \mathrm{~kg} \mathrm{~s}^{-1}$ and a rotating wind is present, as shown in Fig. 3, while the mean and the standard deviation of the initial total grain-size distribution are, respectively, 2 and 1.5, expressed in the $\phi$ scale. The results of the numerical simulations obtained with the three different formulations are presented in Fig. 4 and they perfectly match, showing that the method of moments (dotted lines), both applied to the continuous distribution of the particle number (red) or to the mass distribution (green), gives the same results of the classical formulation based on the discretization of the mass distribution in bins (solid line). For these simulations, we used only the first six moments of the distributions, while 13 bins have been employed with the discretized formulation. This results in a smaller number of equations to solve for the method of moments and, despite the additional cost of the method of moments due to the evaluation of the quadrature points and formulas through the Wheeler algorithm, in a smaller computational time, with a gain of about $30 \%$.

\subsection{Simulation results}

In this section we want to study the variation during the ascent of solid mass flux (due to particle settling) and of the mean and the variance of the mass distribution along the column. As shown in the previous section, there are no significant differences in the results obtained with the three different descriptions of the grain-size distribution. For this reason, in the following we restrict the analysis only to the formula- 

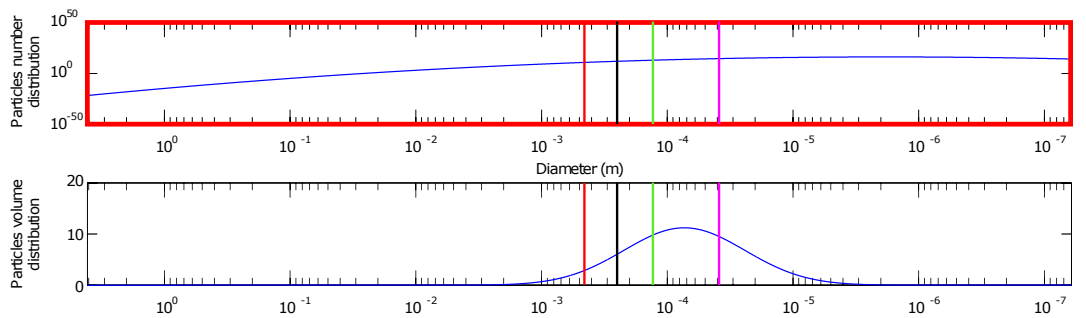

Mean value $\mu$ of the initial mass-fraction distribution expressed as a function of
diameter in the phi scale
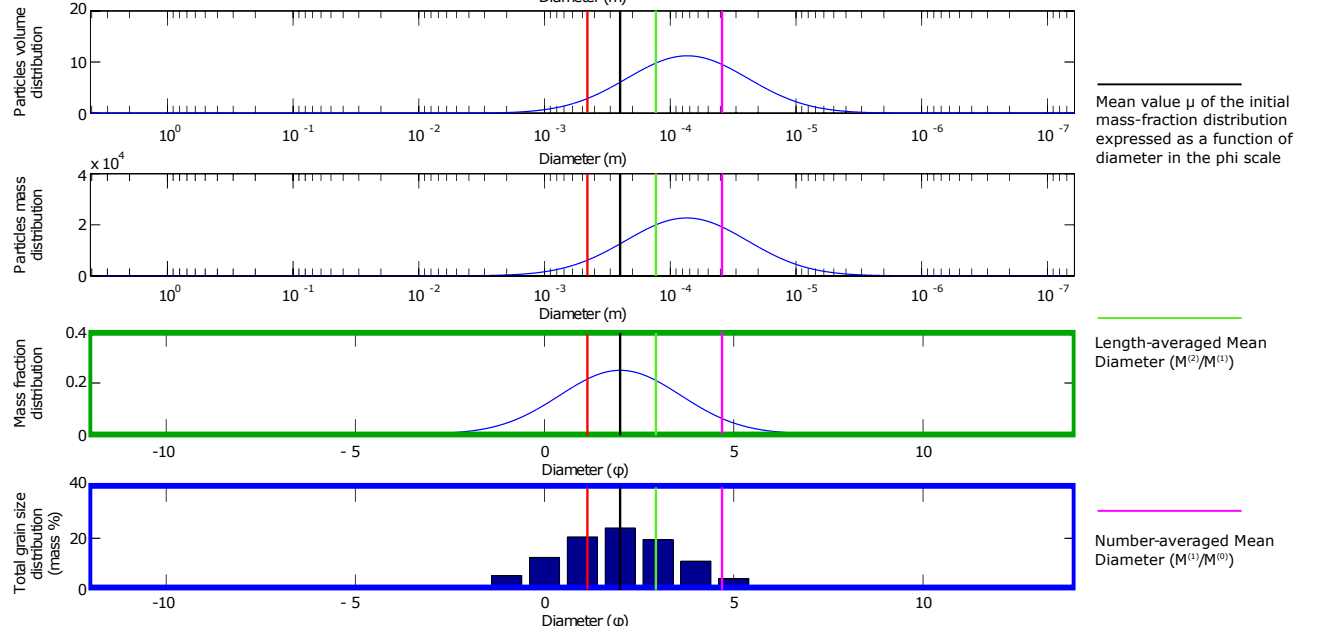
Number-averaged Mean Diameter $\left(\mathrm{M}^{(1)} / \mathrm{M}^{(0)}\right)$

Figure 2. Visualization of a normal initial distribution in the Krumbein $\phi$ scale for the solid particles. On the top plot, the particle number distribution expressed as a function of the diameter expressed in meters is plotted. On the second and third plots from the top, the corresponding distributions of volume and mass are plotted, these two being different because the density is a function of the diameter. On the fourth plot the continuous distribution (lognormal) of mass fraction as a function of the $\phi$ scale is plotted, while in the last plot the distribution has been discretized with 13 bins in the range $(-4 ; 8)$. On each panel different average radii are also plotted, together with the mean of the initial distribution. The first, fourth and fifth panel are highlighted with different colors, also used in Fig. 4 for the solutions obtained with the three different representations of the initial grain-size distribution.
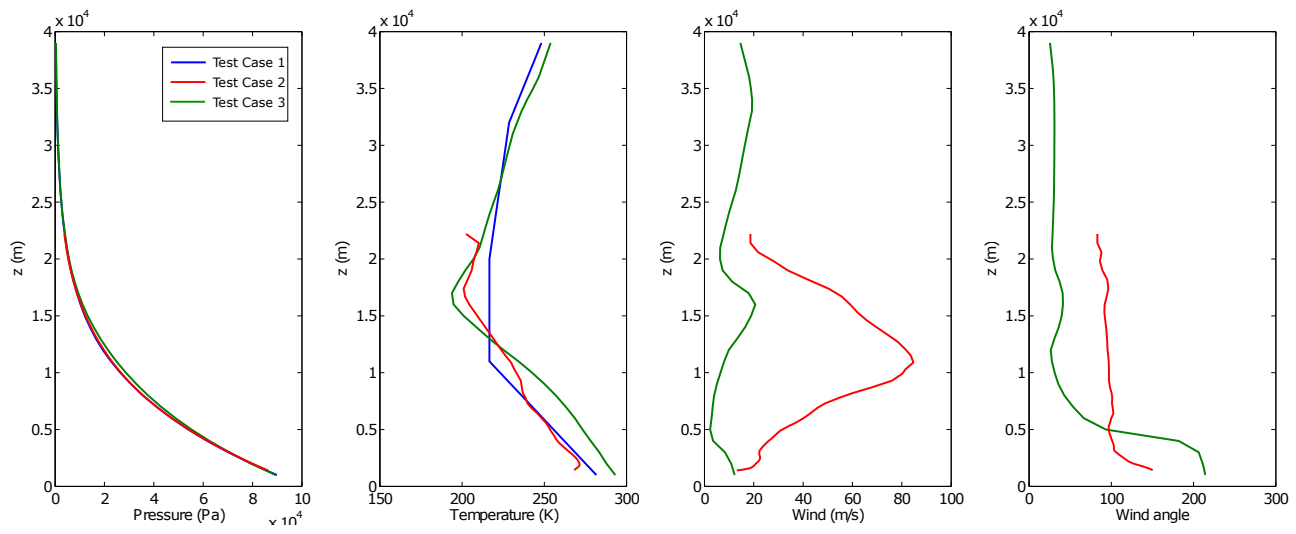

Figure 3. Atmospheric profiles for the three test cases. The height is expressed in meters above sea level, and for all the test cases the vent is located at $1500 \mathrm{~m}$ above sea level. For the wind profiles, only the profiles for the two test cases with wind are plotted.

tion based on the moments of the mass fraction distribution as a function of the diameter expressed in the $\phi$ scale. With this approach, the mean, the variance and the skewness of the mass distribution along the column are easily obtained from the first four moments $\Pi^{(i)}$ of the mass fraction distribution.

In Fig. 5 we present the results relative to the test case 2 for an initial particle size distribution with mean diameter 2 and standard deviation 1.5, expressed in the $\phi$ scale. In the left and middle panels the mean, the variance and the skew of the mass fraction distribution are shown, while in the right panel the cumulative loss of solid mass flux is plotted as a percentage of the initial value. We observe a decrease in the mean size of the particles, due to the different settling velocities of particles of different sizes. A decrease in the variance of the size distribution with height is also observed from the second plot. We remark that the particles have a normal distribution only at the base of the column (resulting in a null skewness), and the negative skew at the top of the column indicates that the tail on the left side of the grain-size distribution is longer than the tail on the right side; i.e., the mass 

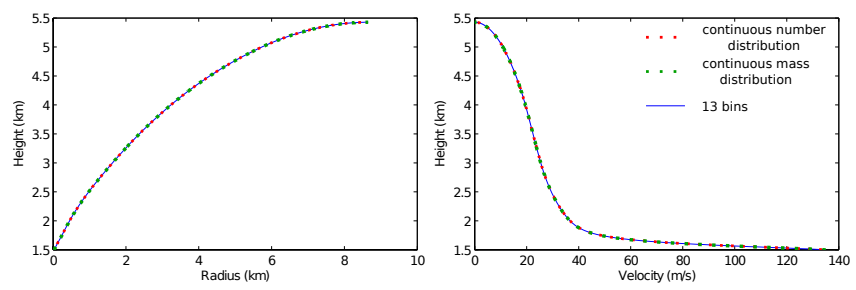

Figure 4. Height vs. radius (left) and velocity (right) for a low-flux plume, simulated with three different models. In blue the profiles obtained using 13 bins, in red the profiles obtained using a continuous distribution of the particle number density and in green using a continuous distribution of the mass fraction.

is more concentrated on the right of the spectrum of particle sizes (finer particles). For this reason we do not have to look at the mean and the variance plotted in Fig. 5 as the parameters of a normal (and symmetric) distribution. Nonetheless, changes in the mean, the variance and the skewness are observed, we remark that these changes are quite small and for this reason the parameters of the total grain-size distribution at the top of the eruptive column are a good approximation of the parameters at the base of the column, and vice versa. However, this is true for the specific input condition of this test case and not in general. For this reason, it is important to quantify the uncertainty of this assumption for different initial total grain-size distributions and different atmospheric conditions.

\subsection{Uncertainty and sensitivity analysis}

When dealing with volcanic processes and volcanic hazards, our understanding of the physical system is limited, and vent parameters (volatile contents, temperature, grain-size distribution, etc.) are often not well constrained or are constrained with significant uncertainty. These factors mean that it is difficult to predict the characteristic of the ash cloud released from the volcanic column with certainty. An alternative is to quantify the probability of the outcomes (for example the grain-size distribution at the top of the column) by coupling deterministic numerical codes with stochastic approaches. It is our goal in this work also to assess the ability to systematically quantify the uncertainty and the sensitivity of the plume model outcomes to uncertain or variable input parameters, in particular to those characterizing the grain-size distribution at the base of the eruptive column.

Uncertainty quantification (UQ) or nondeterministic analysis is the process of characterizing input uncertainties, propagating forward these uncertainties through a computational model, and performing statistical or interval assessments on the resulting responses. This process determines the effect of uncertainties on model outputs or results. In particular, in this work we wanted to investigate for different test cases the uncertainty in four response functions (plume height, solid mass flux lost and mean and variance of the mass fraction
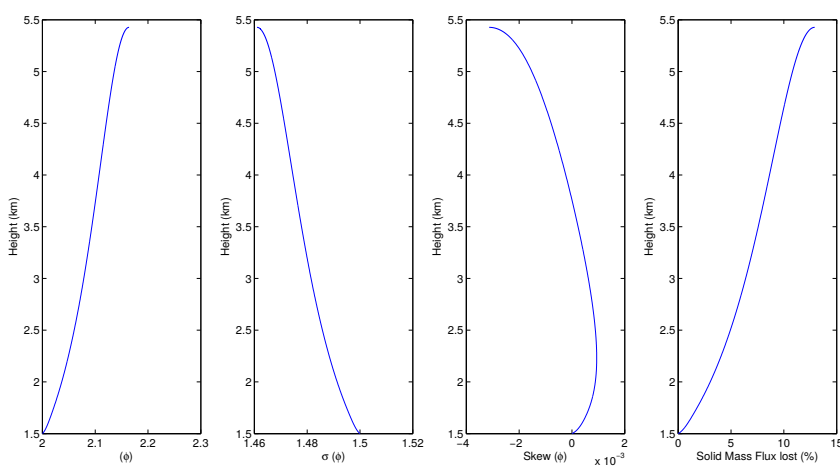

Figure 5. Particle distribution parameters (mean, variance and skewness) and cumulative loss of solid mass flux for the test case 2 (low flux without wind), simulated with the formulation based on the moments of the mass fraction distribution.

distribution at the top of the eruptive column) when the mean and the standard deviation of the distribution at the base are random variables with a uniform probability distribution in the space $(\mu, \sigma) \in[-1 ; 3] \times[0.5 ; 2.5]$.

In volcanology Monte Carlo simulations are frequently used to perform uncertainty quantification analysis. These methods rely on repeated random sampling of input parameters to obtain numerical results; typically one runs simulations many times over in order to obtain the distribution of an unknown output variable. The cost of the Monte Carlo method can be extremely high in terms of number of simulations to run, and thus several alternative approach have been developed. LHS is another sampling technique for which the range of each uncertain variable is divided into $N_{\mathrm{s}}$ segments of equal probability, where $N_{\mathrm{S}}$ is the number of samples requested. The relative lengths of the segments are determined by the nature of the specified probability distribution (e.g., uniform has segments of equal width; normal has small segments near the mean and larger segments in the tails). For each of the uncertain variables, a sample is selected randomly from each of these equal probability segments. These $N_{\mathrm{s}}$ values for each of the individual parameters are then combined in a shuffling operation to create a set of $N_{\mathrm{s}}$ parameter vectors with a specified correlation structure. Compared to Monte Carlo sampling, the LHS has the advantage that in the resulting sample set every row and column in the hypercube of partitions has exactly one sample, and thus a smaller number of samples is required to cover all the parameter space. In the left panel of Fig. 6 an example of LHS with $N_{\mathrm{s}}=10$ and a uniform distribution probability for both $\mu$ and $\sigma$ is plotted.

An alternative approach to uncertainty quantification is the so-called generalized polynomial chaos expansion method, a technique that mirrors deterministic finite element analysis utilizing the notions of projection, orthogonality and weak convergence (Ghanem and Red-Horse, 1999). The polynomial chaos expansion (PCE) method was developed by Norbert Wiener in 1938 and it soon became widely used because 

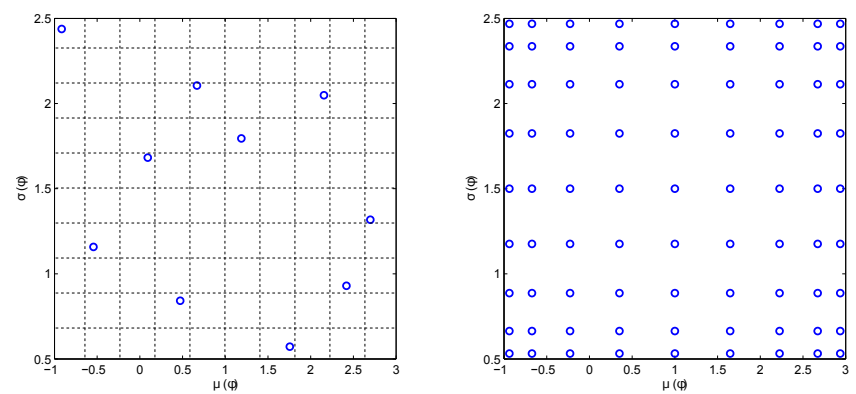

Figure 6. Two-parameters Latin hypercube sampling (LHS) with 10 points (left) and tensor product grid using $9 \times 9$ Clenshaw-Curtis points (right).

of its efficiency when compared to Monte Carlo simulations. The term "chaos" here simply refers to the uncertainties in input, while the word "polynomial" is used because the propagation of uncertainties is described by polynomials. If $\zeta$ is the vector of uncertain input variables, the aim of the gPCE is to express the response function $Y$ in the form of a polynomial $\xi$ as follows:

$\xi(\zeta)=\xi_{0}+\xi_{1} P_{1}(\zeta)+\xi_{2} P_{2}(\zeta)+\ldots+\xi_{m} P_{m}(\zeta)$,

where $P_{1}, \ldots, P_{m}$ are polynomials that form an orthogonal basis. The choice of the polynomials basis depends on the probability distribution of the input variables. In particular, for a uniform distribution, the basis of the expansion is given by the Lagrange polynomials. For the application presented in this work the coefficients of the expansion have been evaluated using a spectral projection where the computation of the required multi-dimensional integrals is based on the tensor product of 1-D Gaussian quadrature rules. In order to compute the quadrature points, the grid used in our work is the Clenshaw-Curtis grid (Fig. 6, right), representing a good solution for a multi-dimensional Gaussian quadrature with a small number of variables (Eldred and Burkardt, 2009).

We present here the results of several tests performed coupling the plume model with the Dakota toolkit (Adams et al., 2009) to investigate systematically the capability of the LHS and the gPCE techniques to assess the uncertainty in four response functions (plume height, solid mass flux lost and mean and variance of the mass fraction distribution at the top of the eruptive column) when the mean and the variance at the base are unknown. For all the test cases three sets of 500, 1000 and 2000 simulations have been performed for the LHS, and the results have been compared with those obtained with three tests for the gPCE and respectively 9, 36 and 81 simulations performed for the multi-dimensional quadrature. The first set of runs for the LHS, consisting of 500 simulations only, was not sufficient to provide accurate results and for this reason in the following we presents only the results obtained with 1000 and 200 simulations. In order to compare the two techniques, the cumulative distributions of the four response functions obtained with the LHS and the gPCE, have been plotted in Fig. 7 for test case 1 (no wind). On the $x$ axes we can see the range of the values obtained for the response functions: $-1-3.5$ for the mean of the total grain-size distribution (TGSD) at top of the column expressed in the $\phi$ scale; $0.4-2.2$ for the standard deviation; $10.41-10.47 \mathrm{~km}$ for the column height and 10-60\% for the percentage of solid mass flux lost. All the uncertainty quantification tests produced very similar results, with a small difference in the cumulative distribution observed only in the distribution of the solid mass flux lost obtained with the gPCE technique and 9 and 36 quadrature points. Similar results have been obtained for the other test cases (not shown here). Thus, the results highlights that for the model and the applications presented in this work gPCE represents a valid alternative to Monte Carlo simulations, with a number of runs required to produce the same accuracy reduced by a factor 10 (81 simulations vs. 1000 simulations). If more parameters were varied, the computational cost would increase for both gPCE and LHS, although the advantage of gPCE would be reduced.

As mentioned previously, the aim of the gPCE is to express the output of the models as polynomials and these polynomials can be used to obtain response surfaces for the output parameters as functions of the unknown input parameters through the polynomials defined by Eq. (54). In the four bottom panels of Fig. 7 the contours of the four response surfaces for the output investigated in this work have been plotted, showing the dependence on the uncertain input parameters. The mean and the standard deviation of the TGSD at the top of the eruptive column clearly reflects the corresponding values at the bottom, with a small effect of the bottom standard deviation on the mean size at the top, resulting in an increase in the average grain size with increasing values of the initial standard deviation (see the curves in the first panel bending on the left for higher values of $\sigma$ ). Conversely, the plume height for this test case shows a nonlinear dependency but at the same time a small sensitivity to the initial grain-size distribution, with changes, for the specific conditions considered here, smaller than $1 \%$ of the average height. This can be explained by the fact that a large amount of air is entrained in the column during the ascent and the contribution of the solid fraction to the overall dynamics becomes small compared to that exerted by the gas. Finally, we observe that the loss of particles is mostly controlled by the mean size of the TGSD.

In Fig. 8 the same contour plots are shown for the polynomial expansion computed for test case 2 (top) and test case 3 (bottom) with 81 quadrature points. The results show again that the total grain-size distribution at the base of the vent represents a reasonable approximation of that at the top of the column. For these test cases, both the column height and the solid mass lost appear to be mostly controlled by the mean size of the TGSD at the base of the column, with a small sensitivity of the height to the initial grain-size distribution. We also observe that the maximum percentage of loss in the solid mass flux is about $15 \%$ for the strong plume simulations, and it is attained for larger mean sizes and smaller variance of the 

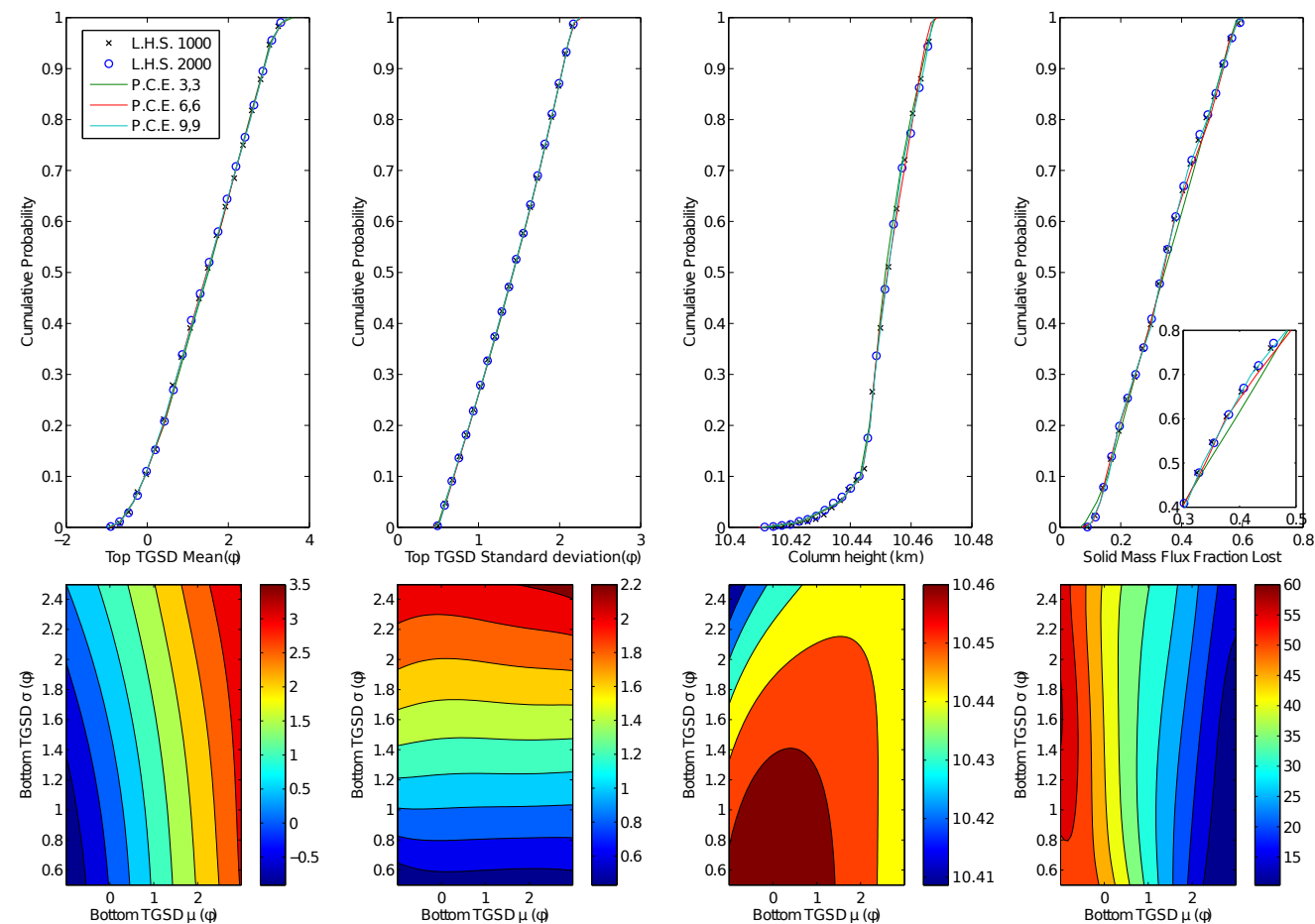

Figure 7. Cumulative distributions and response surfaces for test case 1 (low-flux plume without wind). In the top panels the cumulative probability for several variables describing the outcomes of the simulations (mean and variance of the grain-size distribution at the top of the column, column height and cumulative fraction of solid mass lost) are plotted for the uncertainty quantification analysis carried out with the two different techniques and for different numbers of simulations. The contour plots of the response functions of the four output variables, resulting by the polynomials given by Eq. (54) and obtained with the PCE with 81 quadrature points, are plotted in the bottom panels. The variables contoured in the lower panels are the same as those on the horizontal axes in the upper panels.

initial TGSD. This value is noticeably smaller than that obtained for the weak bent test case $(\approx 40 \%)$ and for the weak test case without wind $(\approx 60 \%)$. Despite the loss of particles, in both the cases the range of variation of the column height is quite small and, as previously mentioned, this is due to the large amount of air entrained in the volcanic column that reduces the contribution of the solid fraction to the overall dynamics. As an example to understand the relevance of the entrained air, for a simulation performed for the low-flux plume without wind and with $\mu=2$ and $\sigma=1.5$ in the $\phi$ scale, the mass flow rate at the top of the column is $1.2 \times 10^{8} \mathrm{~kg} \mathrm{~s}^{-1}$, compared to the value at the base of $1.5 \times 10^{6} \mathrm{~kg} \mathrm{~s}^{-1}$.

\subsection{Sensitivity analysis}

With the polynomial chaos expansion it is also possible to easily obtain the variance-based sensitivity indices (Saltelli et al., 2008) with no additional computational cost. In contrast with some instances, where the term sensitivity is used in a local sense to denote the computation of response derivatives at a point, here the term is used in a global sense to denote the investigation of variability in the response functions. In this context, variance-based decomposition is a global sensitivity method that summarizes how the variability in model output can be apportioned to the variability in individual input variables (Adams et al., 2009). This sensitivity analysis uses two primary measures, the main effect sensitivity index $S_{\mathrm{i}}$ and the total effect index $T_{\mathrm{i}}$. These indices are also called the Sobol indices. The main effect sensitivity index corresponds to the fraction of the uncertainty in the output, $Y$, that can be attributed to input $x_{\mathrm{i}}$ alone. The total effects index corresponds to the fraction of the uncertainty in the output, $Y$, that can be attributed to input $x_{\mathrm{i}}$ and its interactions with other variables. The main effect sensitivity index compares the variance of the conditional expectation $\operatorname{Var}_{x_{\mathrm{i}}}\left[E\left(Y \mid x_{\mathrm{i}}\right)\right]$ against the total variance $\operatorname{Var}(Y)$. Formulas for the indices are

$S_{\mathrm{i}}=\frac{\operatorname{Var}_{x_{\mathrm{i}}}\left[\left(Y \mid x_{\mathrm{i}}\right)\right]}{\operatorname{Var}(Y)}$

and

$T_{\mathrm{i}}=\frac{E\left(\operatorname{Var}\left(Y \mid X_{-\mathrm{i}}\right)\right)}{\operatorname{Var}(Y)}$,

where $Y=f(x)$ and $x_{-\mathrm{i}}=\left(x_{1}, \ldots, x_{\mathrm{i}-1}, x_{\mathrm{i}+1}, \ldots, x_{m}\right)$. Similarly, it is also possible to define the sensitivity indices for higher-order interactions such as the two-way interaction $S_{\mathrm{i}, j}$. The calculation of $S_{\mathrm{i}}$ and $T_{\mathrm{i}}$ requires the evaluation 

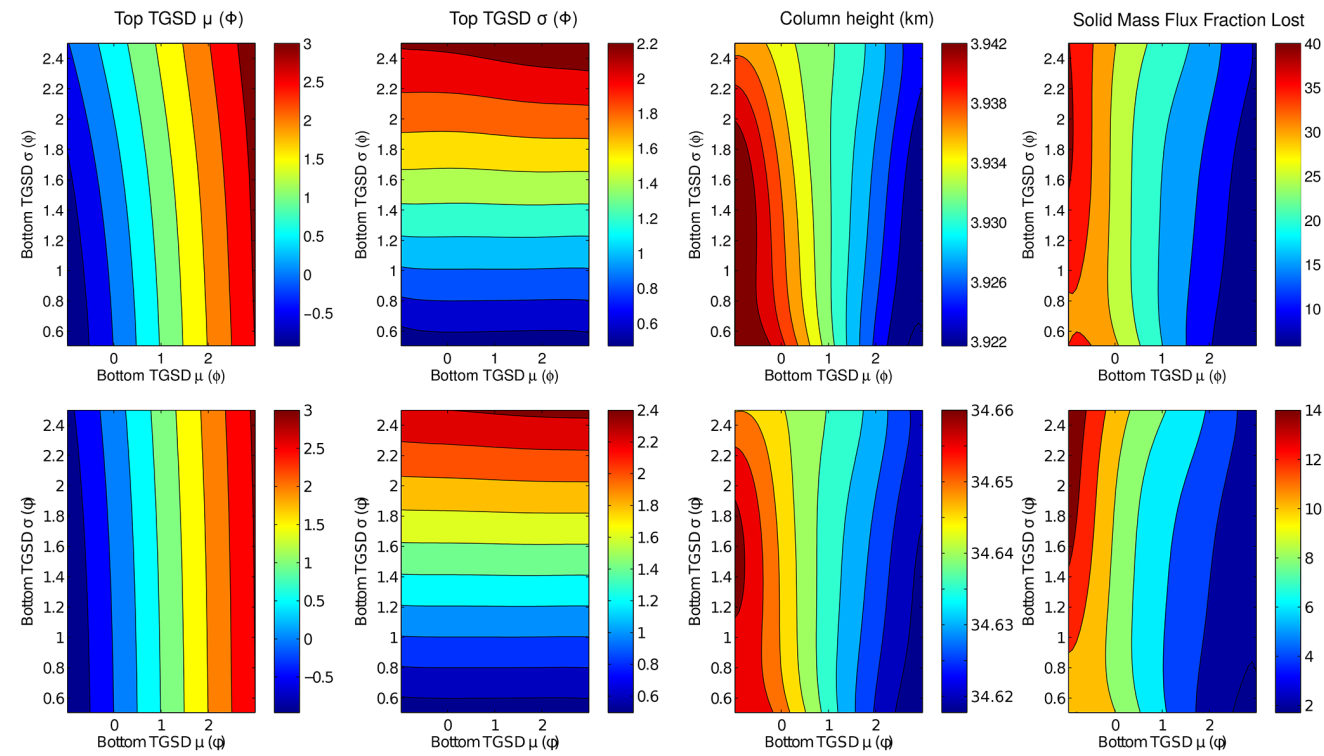

Figure 8. Response surfaces for test case 2 (low-flux plume with wind, four top panels) and test case 3 (strong plume with wind, four bottom panels) obtained with the PCE with 81 quadrature points. Please note that the color scale is not consistent between plots.

of m-dimensional integrals that are typically approximated by Monte Carlo sampling. However, in stochastic expansion methods, it is possible to approximate the sensitivity indices as analytic functions of the coefficients in the stochastic expansion.

The results of the sensitivity analysis for the four outputs and the three test cases investigated are presented in the bar plot of Fig. 9. For each of the four groups (one for each of the different output functions), the three bars represent the main sensitivity indices for the three test cases (test 1 on the left, test 2 in the middle and test 3 on the right), while the different colors are for the sensitivity indices with respect to different variables (blue is for the mean of the initial TGSD, green for the standard variation of the initial TGSD and brown for the second-order coupled interaction). Again, the sensitivity analysis confirms that the mean and the standard deviation of the grain-size distributions at the top of the eruptive column are controlled primarily by the respective parameters of at base of the column. The mean of the TGSD also controls the percentage of solid mass flux lost during the rise of the column and the plume height for the two test cases with wind, while for the weak test case without wind the dispersion of the distribution and second-order interaction also play a major role in controlling plume height variability. However, as already observed with the uncertainty quantification analysis, we remark that the variability in the plume height, when the mean and the standard deviation of the TGSD vary in the investigated ranges, is extremely small for all the test cases (less than $1 \%$ with respect to the average values), and thus the investigation of how the variability in model output can be apportioned to the variability in individual input variables

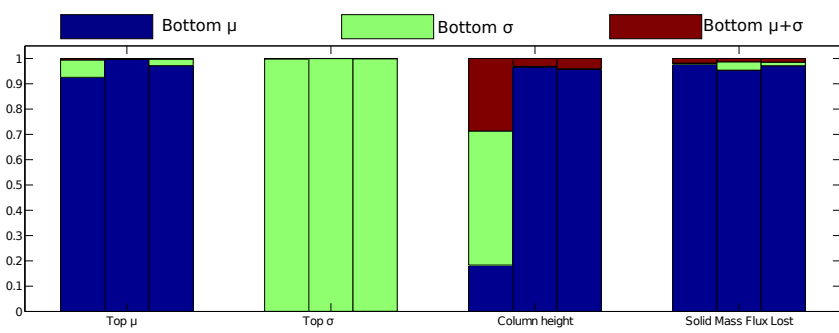

Figure 9. Sobol main sensitivity indices. For each of the four output parameters the three bars are for the different test cases: test case 1 on the left, test case 2 in the middle and test case 3 on the right. For each test case the different colors of the bars are for the different sensitivity indices: blue for first-order sensitivity index with respect to the bottom TGSD mean, green for the first-order sensitivity index with respect to the bottom TGSD standard deviation and brown for the second-order combined sensitivity index.

is less relevant for the plume height than for the other output parameters.

\section{Conclusions}

In this work we have presented an extension, based on the method of moments, of the Eulerian steady-state volcanic plume model presented in Barsotti et al. (2008) (derived from Morton, 1959; Ernst et al., 1996; Bursik, 2001). Two different formulations, one based on a continuous distribution of the number of particles as a function of the size and a second based on the continuous distribution of the mass fraction, have been presented. The tracking of the moments of mass distribution, defined as a function of the Krumbein phi scale, 
has the advantage that with the first three moments only we are able to recover the mean and the standard deviation of the total grain-size distribution. The results of a comparison between the two formulations based on the method of moments and the classical formulation based on the discretization of the mass distribution in bins show that the different approaches produce the same results, with an advantage of the method of moments in terms of computational costs. Furthermore, a formulation based on continuous description of particle size, is better suited to properly describe complex interparticle processes such as particle aggregation and fragmentation that are likely to play an important role in the plume evolution. In particular, the method of moments has already been successfully applied to model aggregation and breakage processes in particulate systems (Marchisio et al., 2003).

An uncertainty quantification analysis has also been applied to the formulation based on the moments of the mass distribution. The results show, for the range of conditions investigated here and neglecting likely relevant interparticle processes such as particle aggregation and comminution, a small change of the mean and variance of the particle mass distribution along the column, indicating that the total grainsize distribution at the base of the vent represents a reasonable approximation of that at the top of the column. Furthermore, based on the plume model assumptions and outcomes, we observe a small sensitivity of the plume height to the initial grain-size distribution, with variations on the order of tens of meters for a plume rising to several kilometers.

For the application presented in this work, involving only two parameters, the comparison between the Latin hypercube sampling technique and the gPCE method shows that the latter only requires 81 simulations to produce the same results, in terms of cumulative probability distributions of several output, obtained with 1000 simulations and the LHS. In fact, the full uncertainty quantification analysis performed on a high-performance computing 48-multicore shared-memory system (HPC-SM) at Istituto Nazionale di Geofisica e Vulcanologia (INGV) in Pisa, Italy, required less than $2 \mathrm{~s}$ for the gPCE method with 81 quadrature points. These results make the new numerical code presented here, coupled with the uncertainty technique investigated, well-suited for realtime hazard assessment.

\section{Code availability}

The source code with the input files for some simulation presented in this work are available for download on the Volcano Modelling and Simulation gateway (http://vmsg.pi.ingv.it/) and on the site for collaborative volcano research and risk mitigation Vhub (https://vhub.org/).

Acknowledgements. The authors are grateful to Samantha Engwell for the helpful discussion and suggestions on the application of the model, and to L. Mastin, Y. Suzuki and M. Woodhouse for their thorough reviews of the manuscript. This work has been partially supported by the project MEDiterranean SUpersite Volcanoes (MED-SUV) FP7 ENV.2012.6.4-2 grant agreement no. 308665 (European Community).

Edited by: J. Williams

\section{References}

Adams, B. M., Bauman, L. E., Bohnhoff, W. J., Dalbey, K. R., Ebeida, M. S., Eddy, J. P., Eldred, M. S., Hough, P. D., Hu, K. T., Jakeman, J. D., Swiler, L. P., and Vigil, D. M.: DAKOTA, A Multilevel Parallel Object-Oriented Framework for Design Optimization, Parameter Estimation, Uncertainty Quantification, and Sensitivity Analysis: Version 5.4 User's Manual, Sandia Technical Report SAND2010-2183, (updated April 2013) 2009.

Barsotti, S., Neri, A., and Scire, J.: The VOL-CALPUFF model for atmospheric ash dispersal: 1. Approach and physical formulation, J. Geophys. Res., 113, B03209, doi:10.1029/2006JB004624, 2008.

Bonadonna, C. and Phillips, J.: Sedimentation from strong volcanic plumes, J. Geophys. Res., 108, 2340, doi:10.1029/2002JB002034, 2003.

Briggs, G. A.: Plume rise predictions, in: Lectures on Air Pollution and Environmental Impact Analyses, edited by: Hangen, D. A., 59-111, American Meteorological Society, Boston, MA, USA, 1975.

Bursik, M.: Effect of wind on the rise height of volcanic plumes, Geophys. Res. Lett, 18, 3621-3624, 2001.

Bursik, M., Sparks, R., Gilbert, J., and Carey, S.: Sedimentation of tephra by volcanic plumes: I. Theory and its comparison with a study of the Fogo A plinian deposit, Sao Miguel (Azores), B. Volcanol., 54, 329-344, 1992.

Carey, S. and Sparks, R. S. J.: Quantitative models of the fallout and dispersal of tephra from volcanic eruption columns, B. Volcanol., 48, 109-125, 1986.

Carneiro, J. N. E.: Development of a Presumed Function Method of Moments with Application to Polydisperse Sprays, PhD Thesis, Technische Universität München, Germany, 2011.

Champion, K., Cole, A., and Kantor, A.: Standard and reference atmospheres Handbook of Geophysics and the Space Environment, Air Force Geophysics Laboratory, 14, Springfield, VA, USA, 1985.

Costa, A., Folch, A., and Macedonio, G.: A model for wet aggregation of ash particles in volcanic plumes and clouds: 1 . Theoretical formulation, J. Geophys. Res.-Sol. Ea., 115, 1978-2012, 2010.

Dartevelle, S.: Numerical modeling of geophysical granular flows: 1. A comprehensive approach to granular rheologies and geophysical multiphase flows, Geochem. Geophy. Geosy., 5, Q08003, doi:10.1029/2003GC000636, 2004.

de' Michieli Vitturi, M., Clarke, A., Neri, A., and Voight, B.: Transient effects of magma ascent dynamics along a geometrically variable dome-feeding conduit, Earth Planet. Sc. Lett., 295, 541553, 2010.

Dufek, J. and Bergantz, G.: Suspended load and bed-load transport of particle-laden gravity currents: the role of particle-bed interaction, Theor. Comp. Fluid Dyn., 21, 119-145, 2007. 
Eldred, M. and Burkardt, J.: Comparison of non-intrusive polynomial chaos and stochastic collocation methods for uncertainty quantification, AIAA paper, 976, 1-20, 2009.

Ernst, G. G., Sparks, R. S. J., Carey, S. N., and Bursik, M. I.: Sedimentation from turbulent jets and plumes, J. Geophys. Res., 101, 5575-5589, doi:10.1029/95JB01900, 1996.

Esposti Ongaro, T., Cavazzoni, C., Erbacci, G., Neri, A., and Salvetti, M. V.: A parallel multiphase flow code for the 3-D simulation of explosive volcanic eruptions, Parallel Comput., 33, 541560, 2007.

Gautschi, W.: Orthogonal polynomials: computation and approximation, Oxford University Press, New York, USA, 2004.

Ghanem, R. and Red-Horse, J. R.: Propagation of probabilistic uncertainty in complex physical systems using a stochastic finite element technique, Physica D, 133, 137-144, 1999.

Glaze, L. S. and Baloga, S. M.: Sensitivity of buoyant plume heights to ambient atmospheric conditions: Implications for volcanic eruption columns, J. Geophys. Res., 101, 1529-1540, 1996.

Golub, G. H. and Welsch, J. H.: Calculation of Gauss quadrature rules, Math. Comput., 23, 221-230, 1969.

Hazewinkel, M.: Moment, Encyclopedia of Mathematics, Springer, the Netherlands, 2001.

Hewett, T., Fay, J., and Hoult, D.: Laboratory experiments of smokestack plumes in a stable atmosphere, Atmos. Environ., 5, 767-789, 1971

Hulburt, H. M. and Katz, S.: Some problems in particle technology: A statistical mechanical formulation, Chem. Eng. Sci., 19, 555574, 1964.

Llewellin, E. W., Mader, H. M., and Wilson, S. D. R.: The constitutive equation and flow dynamics of bubbly magmas, Geophys. Res. Lett., 29, 23-1-23-4, 2002.

Loth, E., O'Brien, T., Syamlal, M., and Cantero, M.: Effective diameter for group motion of polydisperse particle mixtures, Powder Technology, 142, 209-218, doi:10.1016/j.powtec.2004.04.033, 2004.

Marchisio, D. L. and Fox, R. O.: Computational Models for Polydisperse Particulate and Multiphase Systems, Cambridge University Press, New York, USA, 2013.

Marchisio, D. L., Vigil, R. D., and Fox, R. O.: Quadrature method of moments for aggregation-breakage processes, J. Colloid Interf. Sci., 258, 322-334, 2003.

McGraw, R.: Correcting moment sequences for errors associated with advective transport, available at: http://www.ecd.bnl.gov/ pubs/momentcorrection_mcgraw2006.pdf (last access: 5 August 2015), 2006.
Morton, B.: Forced plumes, J. Fluid Mech., 5, 151-163, 1959

Neri, A., Esposti Ongaro, T., Macedonio, G., and Gidaspow, D.: Multiparticle simulation of collapsing volcanic columns and pyroclastic flow , J. Geophys. Res.-Sol. Ea., 108, 1978-2012, 2003.

Pal, R.: Rheological behavior of bubble-bearing magmas, Earth Planet. Sc. Lett., 207, 165-179, 2003.

Petzold, L. R. and Ascher, U. M.: Computer methods for ordinary differential equations and differential-algebraic equations, vol. 61, SIAM, Philadelphia, PA, USA, 1998.

Pfeiffer, T., Costa, A., and Macedonio, G.: A model for the numerical simulation of tephra fall deposits, J. Volcanol. Geoth. Res., 140, 273-294, doi:10.1016/j.jvolgeores.2004.09.001, 2005.

Rietema, K.: The dynamics of fine powders, Springer, the Netherlands, 1991.

Saltelli, A., Ratto, M., Andres, T., Campolongo, F., Cariboni, J., Gatelli, D., Saisana, M., and Tarantola, S.: Global sensitivity analysis: the primer, John Wiley \& Sons, Chichester, West Sussex, England, 2008.

Süli, E. and Mayers, D. F.: An introduction to numerical analysis, Cambridge University Press, Cambridge, UK, 2003.

Textor, C., Graf, H., Herzog, M., Oberhuber, J., Rose, W. I., and Ernst, G.: Volcanic particle aggregation in explosive eruption columns. Part II: Numerical experiments, J. Volcanol. Geoth. Res., 150, 378-394, doi:10.1016/j.jvolgeores.2005.09.008, 2006a.

Textor, C., Graf, H.-F., Herzog, M., Oberhuber, J. M., Rose, W. I., and Ernst, G. G.: Volcanic particle aggregation in explosive eruption columns. Part I: Parameterization of the microphysics of hydrometeors and ash, J. Volcanol. Geoth. Res., 150, 359-377, doi:10.1016/j.jvolgeores.2005.09.007, 2006b.

Valentine, G. A. and Wohletz, K. H.: Numerical Models of Plinian Eruption Columns and Pyroclastic Flows, J. Geophys. Res., 94, 1867-1887, 1989.

Weil, J.: Plume Rise, in: Lectures on Air Pollution Modelling, edited by: Venkatram, A. and Wyngaard, J. C., 119-166, American Meteorological Society, Boston, MA, USA, 1988.

Woods, A. W.: The fluid dynamics and thermodynamics of eruption columns, B. Volcanol., 50, 169-193, doi:10.1007/BF01079681, 1988.

Wright, S. J.: Buoyant jets in density-stratified crossflow, J. Hydraul. Eng., 110, 643-656, 1984. 